\title{
TANGENTIAL EXISTENCE AND COMPARISON, WITH APPLICATIONS TO SINGLE AND MULTIPLE INTEGRATION
}

\author{
BY
}

OLAV KALLENBERG (AUBURN)

\begin{abstract}
Two semi-martingales with respect to a common filtration are said to be tangential if they have the same local characteristics. When the latter are non-random, the underlying semi-martingale is known to have independent increments. We show that every semi-martingale has a tangential process with conditionally independent increments. We also extend the Zinn-Hitchenko and related tangential comparison theorems to continuous time. Combining those results, we obtain some surprisingly general existence, convergence, and tightness criteria for broad classes of single and multiple stochastic integrals.
\end{abstract}

2010 AMS Mathematics Subject Classification: Primary: 60G44, 60G57; Secondary: 60G51, 60H05.

Key words and phrases: Tangential processes, local characteristics, conditional symmetry, marked point processes, compensators, stochastic integration, multiple stochastic integrals.

\section{INTRODUCTION}

Two semi-martingales $X$ and $Y$ with respect to a common filtration $\mathcal{F}$ are said to be tangential if they have the same local characteristics. The latter consist of the quadratic variation of the continuous martingale component, the compensator of the jump point process, and a predictable drift component. Though the definition of the latter is not unique (cf. [9], p. 76), the precise choice is not important for our purposes.

Extending some celebrated theorems of Lévy, Watanabe, and Jacod [6], Grigelionis [2] proved that a semi-martingale $X$ with $X_{0}=0$ has independent increments iff its local characteristics are a.s. non-random (cf. [9], p. 106), in which case the latter determine uniquely the distribution of $X$. This suggests that, for a general semi-martingale $X$, we may reduce to the elementary case of independent increments simply by conditioning on the local characteristics. Unfortunately, that is only possible when $X$ is already a mixture of processes with independent increments, which is not true in general. However, we show in Theorem B.] below that 
every semi-martingale $X$ has a tangential process $Y$ with conditionally independent increments. The idea is then to study the basic properties of $X$ by comparing with the process $Y$, for which the corresponding properties may be elementary and well known.

A closely related notion is that of tangent processes, first mentioned by Itô [4] and featured more prominently in papers by Jacod [ $[7]$ and others. Here we think of the local characteristics $(A, B, C)$ of $X$ as functions of $\omega \in \Omega$. Then for almost every $\omega$ there exists a process $X^{\omega}=\left(X_{t}^{\omega}\right)$ (the tangent process at $\omega$ ) with independent increments and local characteristics $\left(A^{\omega}, B^{\omega}, C^{\omega}\right)$. The previous comparison then amounts to reducing the study of a semi-martingale $X$ to that of its tangent processes $X^{\omega}$.

Related ideas have long been used to extend some classical limit theorems for independent random variables to the dependent case. A more systematic approach was proposed by Jakubowski [10], who identified some general conditioning principles. Some powerful comparison theorems for tangential sequences were obtained by Zinn [27] and Hitchenko [3], and the whole area was explored in monographs by Kwapień and Woyczyński [2T] and de la Peña and Giné [23], which contain extensions, alternative proofs, and numerous applications. In Theorem 4.0 below, we use the approach in [21] to extend the Zinn-Hitchenko inequalities to continuous time.

The tangential comparison gains in power when combined with the classical BDG-inequalities of martingale theory [1]], [12], which allow us to compare martingales that are only weakly tangential, in the sense that the associated quadratic variation processes are strictly tangential. This sometimes leads to the possibility of comparing the original process $X$ with a suitably symmetrized version $\tilde{X}$, an idea also explored in Section 4.

The indicated methods have important applications to stochastic integration, since the basic tangential properties are preserved by integration of predictable processes. Though conditions for integrability and convergence can often be deduced from the general theory of semi-martingales (cf. [12], Chapter 26), the present approach is so much easier whenever it applies, since we only need to know the corresponding criteria for processes with independent increments, where the theory is elementary and accessible by classical methods. Here some results in the independence case are explored in Section 2.1 below, and related criteria for more general stochastic integrals are indicated in Section 5.

A somewhat different approach was developed in [19], [20], where a discretetime tangential comparison is combined with a rather sophisticated approximation argument. Though the resulting criteria are of course equivalent, the results are often stated in terms of Orlicz spaces and other subtle notions of functional analysis.

Multiple stochastic integrals with respect to a Brownian motion were first studied by Itô [5], who was motivated by some earlier work of Wiener (cf. [12], pp. 262ff.). Extensions to more general sequences or processes with independent in- 
crements have been considered by many authors, such as in [16]-[18], [22], [25], [26], where a wide range of methods are employed. In [11], [15], only some elementary properties of Poisson processes were used to find necessary and sufficient conditions for the existence and convergence of multiple integrals with respect to increasing or symmetric Lévy processes. The approach extends easily to any processes with independent, positive or symmetric increments, as indicated in Section 2.2 below.

It has long been an open problem how to apply the tangential methods to multiple integrals with respect to more general processes (cf. Problem 5 in [13]). An obvious first step is to decompose the integral into tetrahedral components (where the coordinates are linearly ordered), which allows us to express its value in terms of iterated single integrations. Since the integrand in each step is predictable, we may now use the results for single stochastic integrals to replace the last (outer) integrator by a tangential process with conditionally independent increments. The problem is that, in order to continue the process recursively, we would need to change the order of integration, which may destroy the predictability of the integrands.

In Section 6 the indicated difficulties are overcome by a subtle trick of conditioning, which leads to some necessary and sufficient conditions for the existence and convergence to zero of multiple integrals with respect to arbitrary marked point processes. (The extension to increasing processes is now routine.) The given criteria, stated in terms of certain sequential compensators with associated sequentially tangential processes, extend the more elementary decoupling relations for Poisson integrals, derived by different methods in [15]. In the more difficult signed case, we can only prove some weaker one-sided results.

Our terminology and notation follow [12]. Thus, we take all processes $X, Y, \ldots$ to be adapted to an underlying filtration $\mathcal{F}=\left(\mathcal{F}_{t}\right)$, satisfying the usual conditions of right-continuity and completeness. For semi-martingales $X$, we denote the associated quadratic variation process by $[X]$. The maximum process is denoted by $X_{t}^{*}=\sup _{s \leqslant t}\left|X_{s}\right|$, and we put $X^{*}=X_{\infty}^{*}$, as usual. For suitable measures $\mu$ and measurable functions $f$, we denote the associated integral by $\mu f=\int f d \mu=$ $\int f_{s} \mu(d s)$, whereas the measure with density $f$ is denoted by $f \cdot \mu$. When the underlying space is $\mathrm{R}_{+}=[0, \infty)$, we may also take $f \cdot \mu$ to mean the function $(f \cdot \mu)_{t}=\int_{0}^{t} f d \mu$, where the integration extends over the semi-open interval $(0, t]$. A similar notation applies to stochastic integrals of predictable processes $V$ with respect to semi-martingales $X$.

We write $\mathrm{N}=\{1,2, \ldots\}$, and let $2^{J}$ or $2^{d}$ denote the class of subsets of $J$ or $\{1, \ldots, d\}$, respectively. The cardinality of the set $J$ is denoted by $|J|$. For any functions $f$ or measures $\mu$, we write $\Delta f_{s}$ or $\Delta \mu_{s}$ for the jump size or point mass at $s$. A relation $f \leq g$ between the functions $f, g \geqslant 0$ means that $f \leqslant c g$ for some constant $c \in(0, \infty)$, and by $f \asymp g$ we mean that both $f \leq g$ and $g \leq f$. The norm $\|\cdot\|$ denotes total variation or supremum, depending on the context. 
For any measurable spaces $S$ and $T$, the associated $\sigma$-fields are denoted by $\mathcal{S}$ and $\mathcal{T}$. Though most results have versions for arbitrary Borel spaces, we may usually take $S$ and $T$ to be separable and complete metric spaces with associated Borel $\sigma$-fields. The unit mass at $s \in S$ is denoted by $\delta_{s}$, whereas the indicator function of the set $B \in \mathcal{S}$ is denoted by $1_{B}$, so that $1_{B}(s)=\delta_{s} B$. For complicated sets $B=\{\cdot\}$, we often write $1_{B}=1\{\cdot\}$, for typographical convenience. Expected values are written as $E \xi$ without brackets, and $\mathcal{L}(\xi)$ denotes the distribution of the random element $\xi$. Independence is denoted by $\Perp$, and conditional independence given $\xi$ by $\Perp_{\xi}$.

\section{PROCESSES WITH INDEPENDENT INCREMENTS}

Since the tangent processes of a general semi-martingale are processes with independent increments, it is important to understand the latter. Here the classical Lévy-Itô representation provides a unique decomposition of such a process $X$ into a Brownian motion of variable rate, a sum of suitably compensated jumps, and a deterministic drift component (cf. [9], [12]). The jump component is best understood in terms of the associated jump point process $\xi$ on $(0, \infty) \times(R \backslash\{0\})$, given for measurable $B \subset \mathrm{R} \backslash\{0\}$ by

$$
\xi([0, t] \times B)=\sum_{s \leqslant t} 1_{B}\left(\Delta X_{s}\right), \quad t \geqslant 0,
$$

which inherits the independence property (in the time variable) from $X$.

More generally, we may consider point processes $\xi$ on $S$ with marks in $T$, for any separable and complete metric spaces $S$ and $T$. Thus, $\xi$ is an integer-valued random measure on $S \times T$, such that $\xi B<\infty$ a.s. for all bounded, measurable sets $B \subset S \times T$, and $\sup _{s} \xi(\{s\} \times T) \leqslant 1$ a.s. By the Lévy-Itô theorem, $\xi$ has independent increments, in the sense that $\xi\left(I_{1} \times \cdot\right), \ldots, \xi\left(I_{n} \times \cdot\right)$ are independent for disjoint $I_{1}, \ldots, I_{n}$, iff

$$
\xi=\eta+\sum_{k}\left(\delta_{s_{k}} \otimes \delta_{\tau_{k}}\right) \text { a.s. }
$$

for some Poisson process $\eta$ on $S \times T$ with $E \eta(\{s\} \times T) \equiv 0$, some distinct points $s_{1}, s_{2}, \ldots \in S$, and some independent random elements $\tau_{1}, \tau_{2}, \ldots$ in $T^{\Delta}=T \cup$ $\{\Delta\}$ with arbitrary $\Delta \notin T$ (cf. Theorem 12.10 in [12]). Writing $\alpha=E \eta$ and $\beta_{k}=$ $\mathcal{L}\left(\tau_{k}\right)$, we note that $\xi$ has intensity measure

$$
\nu=\alpha+\sum_{k}\left(\delta_{s_{k}} \otimes \beta_{k}\right)=\alpha+\sum_{k} \nu_{k}
$$

which is locally finite on $S \times T$ and determines uniquely the distribution of $\xi$. Conversely, any such measure $\nu$ on $S \times T$ with $\sup _{s} \nu(\{s\} \times T) \leqslant 1$ may qualify. Note that $\xi$ is Poisson iff it has no fixed discontinuities, in the sense that $\nu(\{s\} \times T) \equiv 0$. 
2.1. Single integrals. Using elementary formulas for Laplace transforms, we can easily derive criteria for $\xi_{n} f_{n} \stackrel{P}{\rightarrow} 0$ or $\infty$ when the $\xi_{n}$ are Poisson processes on $S$ with arbitrary intensities $E \xi_{n}=\nu_{n}$. Here we consider the more general case of marked point processes $\xi_{n}$ with independent increments. For the easy proofs, we refer to [14]. Define $\psi(x)=1-e^{-x}$.

LEMMA 2.1. Let $\xi, \xi_{1}, \xi_{2}, \ldots$ be T-marked point processes on $S$ with independent $S$-increments and intensities $\nu, \nu_{1}, \nu_{2}, \ldots$ Then for any functions $f, f_{1}, f_{2}, \ldots \geqslant 0$ on $S \times T$,

(i) $P\{\xi f<\infty\}=1\{\nu(f \wedge 1)<\infty\}$;

(ii) $\xi_{n} f_{n} \stackrel{P}{\rightarrow} 0$ iff $\nu_{n}\left(f_{n} \wedge 1\right) \rightarrow 0$; left when

(iii) $\nu_{n}\left(f_{n} \wedge 1\right) \rightarrow \infty \Rightarrow \xi_{n} f_{n} \stackrel{P}{\rightarrow} \infty \Rightarrow \nu_{n} f_{n} \rightarrow \infty$, with equivalence on the

$$
\limsup _{n \rightarrow \infty}\left(\left\|\Delta \nu_{n}\right\| \wedge\left\|\psi \circ f_{n}\right\|\right)<1
$$

We turn to the corresponding criteria for integrals of symmetric point processes. Given a simple point process $\xi$ with intensity $\nu$, we may write $\xi=\xi^{\prime}+\xi^{\prime \prime}$, where $\xi^{\prime}$ and $\xi^{\prime \prime}$ are complementary $\frac{1}{2}$-thinnings of $\xi$ with intensity $\nu / 2$. The symmetrized version $\tilde{\xi}$ of $\xi$ may then be defined as $\xi^{\prime}-\xi^{\prime \prime}$. Equivalently, we may form $\tilde{\xi}$ by attaching independent, symmetric signs to the atoms of $\xi$.

The integral $\tilde{\xi} f$ is elementary when the support of $f$ has finite $\nu$-measure. In general, it is defined by the condition $\tilde{\xi} f_{n} \stackrel{P}{\rightarrow} \tilde{\xi} f$ for any functions $f_{n} \rightarrow f$ with bounded support satisfying $\left|f_{n}\right| \leqslant|f|$, whenever the limit exists and is a.s. independent of the choice of approximating sequence $\left(f_{n}\right)$. The proof of statement (iii) below may be based on the Kolmogorov-Rogozin inequality (cf. [24], p. 68).

LEMMA 2.2. Let $\tilde{\xi}, \tilde{\xi}_{1}, \tilde{\xi}_{2}, \ldots$ be symmetric, $T$-marked point processes on $S$ with independent $S$-increments and intensities $\nu, \nu_{1}, \nu_{2}, \ldots$ Then for any functions $f, f_{1}, f_{2}, \ldots$ on $S \times T$,

(i) $\tilde{\xi} f$ exists iff $\nu\left(f^{2} \wedge 1\right)<\infty$, and when $S=\mathrm{R}_{+}$, we have

$$
P\left\{(f \cdot \tilde{\xi})^{*}<\infty\right\}=1\left\{\nu\left(f^{2} \wedge 1\right)<\infty\right\} ;
$$

(ii) $\tilde{\xi}_{n} f_{n} \stackrel{P}{\rightarrow} 0$ iff $\nu_{n}\left(f_{n}^{2} \wedge 1\right) \rightarrow 0$;

(iii) $\nu_{n}\left(f_{n}^{2} \wedge 1\right) \rightarrow \infty \Rightarrow\left|\tilde{\xi}_{n} f_{n}\right| \stackrel{P}{\rightarrow} \infty \Rightarrow \nu_{n} f_{n}^{2} \rightarrow \infty$, with equivalence on the left when

$$
\limsup _{n \rightarrow \infty}\left(\left\|\Delta \nu_{n}\right\| \wedge\left\|\psi \circ f_{n}^{2}\right\|\right)<1
$$

Given a simple point process $\xi$ with independent increments and intensity $\nu$, we may next consider the compensated integrals $(\xi-\nu) f$, defined by the condition $(\xi-\nu) f_{n} \stackrel{P}{\rightarrow}(\xi-\nu) f$ for bounded functions $f_{n} \rightarrow f$ with bounded supports 
and $\left|f_{n}\right| \leqslant|f|$, whenever the limit exists and is a.s. independent of the choice of approximating sequence $\left(f_{n}\right)$. To avoid near cancellations, we need to assume

$$
\sup _{s \in S} \nu\{s\}<1, \quad \sup _{n \geqslant 1} \sup _{s \in S} \nu_{n}\{s\}<1 .
$$

LEMMA 2.3. Let $\xi, \xi_{1}, \xi_{2}, \ldots$ be simple point processes on $S$ with independent increments and intensities $\nu, \nu_{1}, \nu_{2}, \ldots$ satisfying (2.5). Then for any functions $f, f_{1}, f_{2}, \ldots \geqslant 0$ on $S$ have

(i) $(\xi-\nu) f$ exists iff $\nu\left(f^{2} \wedge f\right)<\infty$, and for $S=\mathrm{R}_{+}$and bounded $f$ we

$$
P\left\{(f \cdot(\xi-\nu))^{*}<\infty\right\}=1\left\{\nu f^{2}<\infty\right\} ;
$$

(ii) $\left(\xi_{n}-\nu_{n}\right) f_{n} \stackrel{P}{\rightarrow} 0$ iff $\nu_{n}\left(f_{n}^{2} \wedge f_{n}\right) \rightarrow 0$.

We also consider the intermediate case between the symmetric and compensated integrals in Lemmas 2.2 and 2.3 .

COROLlARY 2.1. Let $\xi^{ \pm}, \xi_{1}^{ \pm}, \xi_{2}^{ \pm}, \ldots$ be pairwise independent, simple point processes on $S$ with independent increments and intensities $\nu^{ \pm}, \nu_{1}^{ \pm}, \nu_{2}^{ \pm}, \ldots$, satisfying conditions as in (2.5)), and put $\xi=\xi^{+}-\xi^{-}, \nu=\nu^{+}-\nu^{-}$, and $|\nu|=$ $\nu^{+}+\nu^{-}$, and similarly for $\xi_{n}, \nu_{n}$, and $\left|\nu_{n}\right|$. Then for any uniformly bounded functions $f, f_{1}, f_{2}, \ldots$ on $S$,

(i) $(\xi-\nu) f$ exists iff $|\nu| f^{2}<\infty$, and when $S=\mathrm{R}_{+}$, we have

$$
P\left\{(f \cdot(\xi-\nu))^{*}<\infty\right\}=1\left\{|\nu| f^{2}<\infty\right\} ;
$$

(ii) $\left(\xi_{n}-\nu_{n}\right) f_{n} \stackrel{P}{\rightarrow} 0$ iff $\left|\nu_{n}\right| f_{n}^{2} \rightarrow 0$.

For subsequent needs, we show how in the previous results we may add some independent Gaussian random variables to the symmetric or compensated point process integrals $\tilde{\xi}_{n} f_{n}$ or $\left(\xi_{n}-\nu_{n}\right) f_{n}$. We state the resulting extension only for Lemma 2.2 (ii), the remaining cases being similar.

COROLlaRY 2.2. For each $n \in \mathrm{N}$, let $\tilde{\xi}_{n}$ be a symmetric, T-marked point process on $S$ with independent $S$-increments and intensity $\nu_{n}$, and let $\gamma$ be an independent $N(0,1)$ random variable. Then for any functions $f_{n}$ on $S \times T$ and constants $\sigma_{n}$,

$$
\tilde{\xi}_{n} f_{n}+\sigma_{n} \gamma \stackrel{P}{\rightarrow} 0 \quad \Leftrightarrow \quad \nu_{n}\left(f_{n}^{2} \wedge 1\right)+\sigma_{n}^{2} \rightarrow 0 .
$$

For suitable processes on $\mathrm{R}_{+}$, the previous results extend to criteria for uniform boundedness and convergence.

COROLLARY 2.3. Let $X, X_{1}, X_{2}, \ldots$ be centered processes with independent increments, such that the jumps of modulus greater than one are symmetric or have positive compensator. Then 
(i) $\left|X_{\infty}\right|<\infty$ a.s. $\Leftrightarrow X^{*}<\infty$ a.s.

(ii) $X_{n}(\infty) \stackrel{P}{\rightarrow} 0 \Leftrightarrow X_{n}^{*} \stackrel{P}{\rightarrow} 0$.

In general, we say that the process $X$ or sequence $\left(X_{n}\right)$ satisfies the maximum property if equivalence holds in (i) or (ii).

2.2. Multiple integrals. A fairly complete theory of multiple Poisson integrals was developed in [15]. Most results extend with essentially the same proofs to the more general setting of processes with independent increments, and most results for symmetric integrals remain valid when the underlying processes $\xi$ and $\xi_{1}, \ldots, \xi_{d}$ are completely arbitrary. For those reasons we will only quote without proofs some of the main results, often in simplified form, for subsequent comparison and reference. A fuller treatment can be found in [14].

First let $\xi$ and $\xi_{1}, \ldots, \xi_{d}$ be i.i.d. $T$-marked point processes on $S$ with intensity $\nu$, and put $\bar{S}=S \times T$. Given any function $f \geqslant 0$ on $\bar{S}^{J}$ with $|J|<\infty$, we define recursively some functions $f_{1}, \ldots, f_{|J|} \geqslant 0$ on $\bar{S}^{J}$ by

$$
f_{1}=f \wedge 1, \quad f_{k+1}=f_{k} \prod_{|I|=k} 1\left\{\nu^{I} f_{k} \leqslant 1\right\}, \quad 1 \leqslant k<|J|,
$$

where the product extends over all sets $I \subset J$ with $|I|=k$, and $\nu^{I}$ denotes integration in the arguments indexed by $I$, so that $\nu^{I} f_{k}$ becomes a measurable function of the remaining arguments indexed by $J \backslash I$.

Next define recursively some classes $\mathcal{C}_{d}$ of measurable functions $f \geqslant 0$ on $\bar{S}^{d}$. Starting with $\mathcal{C}_{0}=\{0,1\}$ and assuming $\mathcal{C}_{k}$ to be given for all $k<d$, we define $\mathcal{C}_{d}$ by the conditions

$$
\left\{\begin{array}{l}
\nu^{d-k}\left\{\nu^{J} f_{k}=\infty\right\}=0, \\
1\left\{\nu^{J} f_{k}>1\right\} \in \mathcal{C}_{d-k},
\end{array} \quad J \in 2^{d} \text { with } k=|J|>0 .\right.
$$

We may now characterize the a.s. finiteness and convergence to zero of the multiple integrals $\xi_{1} \ldots \xi_{d} f$ and $\xi^{d} f$. For notational convenience, we consider only the case of constant $\nu$. Given some measurable functions $f_{n} \geqslant 0$ on $\bar{S}^{d}$, we define the associated truncated functions $f_{n}^{1}, \ldots, f_{n}^{d}$ as in (2.6). Say that a function $f$ on $S^{d}$ is non-diagonal if it vanishes on all diagonal spaces where two or more variables agree.

Lemma 2.4. Let $\xi, \xi_{1}, \ldots, \xi_{d}$ be i.i.d. T-marked point processes on $S$ with independent increments and intensity $\nu$, and let $f, f_{1}, f_{2}, \ldots \geqslant 0$ be measurable functions on $\bar{S}^{d}$. Then

(i) $P\left\{\xi_{1} \ldots \xi_{d} f<\infty\right\}=1\left\{f \in \mathcal{C}_{d}\right\}$;

(ii) $\xi_{1} \ldots \xi_{d} f_{n} \stackrel{P}{\rightarrow} 0$ iff $\nu^{d} f_{n}^{d} \rightarrow 0$ and

$$
\xi_{1} \ldots \xi_{d-k}\left\{\nu^{J} f_{n}^{k}>1\right\} \stackrel{P}{\rightarrow} 0, \quad J \in 2^{d} \text { with } k=|J|>0 .
$$


Both results remain true for the integrals $\xi^{d} f$ and $\xi^{d} f_{n}$ when $f$ and all $f_{n}$ are non-diagonal.

Next let $\zeta$ and $\zeta_{1}, \ldots, \zeta_{d}$ be conditionally independent symmetrizations of some simple point processes $\xi$ and $\xi_{1}, \ldots, \xi_{d}$ on $S$. Thus, if $\xi=\sum_{k} \delta_{\tau_{k}}$, we may define $\zeta=\sum_{k} \sigma_{k} \delta_{\tau_{k}}$, where the random signs $\sigma_{k}$ are i.i.d. and independent of $\tau_{1}, \tau_{2}, \ldots$ with distribution $P\left\{\sigma_{k}= \pm 1\right\}=\frac{1}{2}$. For $\xi_{1}, \ldots, \xi_{d}$ we need the associated sign sequences $\sigma_{1}, \ldots, \sigma_{d}$ to be independent.

For any measurable function $f$ on $S^{d}$, we define $\zeta_{1} \ldots \zeta_{d} f$ as the limit in probability of the elementary integrals $\zeta_{1} \ldots \zeta_{d} f_{n}$, for functions $f_{n} \rightarrow f$ with bounded supports satisfying $\left|f_{n}\right| \leqslant|f|$, provided that the limit exists and is a.s. independent of the choice of $f_{1}, f_{2}, \ldots$ The definition of the integral $\zeta^{d} f$ is similar.

In the special case where $\xi$ and $\xi_{1}, \ldots, \xi_{d}$ are equal to counting measure on $\mathrm{N}$, the integrals reduce to multi-linear forms in $\sigma$ or $\sigma_{1}, \ldots, \sigma_{d}$, here denoted by $\sigma^{d} A$ or $\sigma_{1} \ldots \sigma_{d} A$ for suitable arrays $A=\left(a_{k}\right)$ indexed by $\mathrm{N}^{d}$. For general point processes $\xi=\sum_{k} \delta_{\tau_{k}}$ on $S$ and functions $f$ on $S^{d}$, we define $f\left(\xi^{d}\right)$ as the array on $\mathrm{N}^{d}$ with entries $f\left(\tau_{k_{1}}, \ldots, \tau_{k_{d}}\right)$. The definition of $f\left(\xi_{1} \ldots \xi_{d}\right)$ is similar.

We may now state the basic existence and representation theorem for multiple symmetric integrals.

LeMmA 2.5. Let $\xi$ and $\xi_{1}, \ldots, \xi_{d}$ be simple point processes on $S$ with symmetrizations $\zeta$ and $\zeta_{1}, \ldots, \zeta_{d}$, generated by the independent sign sequences $\sigma$ and $\sigma_{1}, \ldots, \sigma_{d}$, and fix any measurable function $f$ on $S^{d}$. Then

(i) the integral $\zeta_{1} \ldots \zeta_{d} f$ exists iff $\xi_{1} \ldots \xi_{d} f^{2}<\infty$ a.s., and similarly for the integrals $\zeta^{d} f$ and $\xi^{d} f^{2}$ when $f$ is symmetric non-diagonal;

(ii) the following representations hold a.s. whenever either side exists:

$$
\zeta_{1} \ldots \zeta_{d} f=\left(\sigma_{1} \ldots \sigma_{d}\right) f\left(\xi_{1} \ldots \xi_{d}\right), \quad \zeta^{d} f=\sigma^{d} f\left(\xi^{d}\right) .
$$

In other words, the two sides exist simultaneously, in which case they are equal. By independence of the sign sequences, we may form the multi-linear forms on the right by first conditioning on the processes $\xi$ and $\xi_{1}, \ldots, \xi_{d}$.

We finally compare the various integrals considered above, under appropriate conditions on the integrands and underlying point processes.

COROLlARY 2.4. Let $\xi$ and $\xi_{1}, \ldots, \xi_{d}$ be simple point processes on $S$ with conditionally independent symmetrizations $\zeta$ and $\zeta_{1}, \ldots, \zeta_{d}$, and let $f, f_{1}, f_{2}, \ldots$ be measurable functions on $S^{d}$. Then

(i) $\zeta_{1} \ldots \zeta_{d} f$ exists iff $\xi_{1} \ldots \xi_{d} f^{2}<\infty$ a.s.;

(ii) $\zeta_{1} \ldots \zeta_{d} f_{n} \stackrel{P}{\rightarrow} 0$ iff $\xi_{1} \ldots \xi_{d} f_{n}^{2} \stackrel{P}{\rightarrow} 0$.

If $f, f_{1}, f_{2}, \ldots$ are symmetric non-diagonal, then also

(iii) $\zeta^{d} f$ exists iff $\xi^{d} f^{2}<\infty$ a.s.;

(iv) $\zeta^{d} f_{n} \stackrel{P}{\rightarrow} 0$ iff $\xi^{d} f_{n}^{2} \stackrel{P}{\rightarrow} 0$. 
If $\xi$ and $\xi_{1}, \ldots, \xi_{d}$ are i.i.d. T-marked on $S$ with independent increments and $f, f_{1}, f_{2}, \ldots$ are symmetric non-diagonal on $\bar{S}^{d}$, then the conditions in (i) and (ii) are equivalent to the corresponding ones in (iii) and (iv).

\section{TANGENTIAL EXISTENCE AND RANDOMIZATION}

Let $\mathcal{F}=\left(\mathcal{F}_{t}\right)$ be a filtration on $\mathrm{R}_{+}$satisfying the usual conditions of rightcontinuity and completeness. Given an $\mathcal{F}$-adapted, $T$-marked point process $\xi$ on $(0, \infty)$, we define the $\mathcal{F}$-compensator of $\xi$ as the a.s. unique predictable random measure $\hat{\xi}$ on $\mathrm{R}_{+} \times T$ such that $E \xi V=E \hat{\xi} V$ for all predictable processes $V \geqslant 0$ on $\mathrm{R}_{+} \times T$. Note that $\hat{\xi}$ always exists, by Theorem 25.22 in [12]. Say that $\xi$ has $\mathcal{F}$-independent increments if $\theta_{t} \xi \Perp \mathcal{F}_{t}$ for all $t \geqslant 0$, where $\left(\theta_{t} \xi\right) B=\xi(B+t)$ for measurable $B \subset(0, \infty) \times T$, and $\Perp$ denotes independence. By a fundamental theorem of Jacod (cf. [9], p. 106), $\xi$ has $\mathcal{F}$-independent increments iff $\hat{\xi}$ is a.s. nonrandom, in which case $\xi$ has intensity $E \xi=\hat{\xi}$. In the ql-continuous (quasi-leftcontinuous) case, the process $\xi$ is then Poisson, and the result reduces to Watanabe's theorem.

Given a real semi-martingale $X$, we define the associated jump point process $\xi$ on $\mathrm{R}_{+} \times(\mathrm{R} \backslash\{0\})$ by (2.1) and write $\hat{\xi}$ for the compensator of $\xi$. If $X$ is increasing with $X_{0}=0$, we define the local characteristics of $X$ as the pair $\left(X^{c}, \hat{\xi}\right)$, where $X^{c}$ denotes the continuous component of $X$. For local martingales $X$ with $X_{0}=0$, the local characteristics are instead defined as the pair $\left([X]^{c}, \hat{\xi}\right)$, where $[X]^{c}$ denotes the continuous component of the quadratic variation $[X]$ of $X$.

Two semi-martingales $X$ and $Y$, adapted to a common filtration $\mathcal{F}$, are said to be tangential if their local characteristics agree a.s. We also say that two local martingales $X$ and $Y$ are weakly tangential if their increasing processes $[X]$ and $[Y]$ are tangential. The latter property is indeed weaker than the proper tangential relation between $X$ and $Y$. Our main result in this section is Theorem B.I, which shows that any semi-martingale $X$ has a tangential process $Y$ with conditionally independent increments. In general, this is only true after a suitable extension of the underlying filtration $\mathcal{F}$. Given two filtrations $\mathcal{F}$ and $\mathcal{G}$ on a common probability space $\Omega$, we say that $\mathcal{G}$ is a standard extension of $\mathcal{F}$ if

$$
\mathcal{F}_{t} \subset \mathcal{G}_{t} \Perp_{\mathcal{F}_{t}} \mathcal{F}, \quad t \geqslant 0
$$

where the second relation means that $G \Perp_{\mathcal{F}_{t}} F$ for all $G \in \mathcal{G}_{t}$ and $F \in \bigcup_{u} \mathcal{F}_{u}$. This is precisely the condition needed for all adaptedness and conditioning properties to be preserved (cf. [12], p. 352).

A discrete-time version of the mentioned result is well known and due to Kwapień and Woyczyński [20] (cf. [23], pp. 293f., or [21], pp. 103ff.). In fact, it is worth pointing out that all major results in this paper have discrete-time versions, which follow as special cases of the continuous-time statements (but not 
the other way around). To see this, it suffices to embed the discrete-time scale $Z_{+}$ into $\mathrm{R}_{+}$and extend the discrete filtration $\mathcal{F}=\left(\mathcal{F}_{k}\right)$ to $\mathrm{R}_{+}$by setting $\mathcal{F}_{t}=\mathcal{F}_{[t]}$ for all $t \geqslant 0$, where $[t]$ denotes the integral part of $t$. All tangential and predictable properties of random sequences $X=\left(X_{k}\right)$ remain valid for the continuous-time extensions $X_{t}=X_{[t]}$.

We should also comment on the relationship between the present results and those of Jacod and Sadi [8], where the authors identify a broad class of processes $X$ containing all semi-martingales, such that $X$ admits a tangent process with conditionally independent increments. This is understood in the sense of convergence in probability of certain random probability measures on the Skorokhod space $D\left(\mathrm{R}_{+}\right)$, constructed from sums of conditional probabilities associated with $X$. We may think of the limiting random measure as the conditional distribution of the desired tangent process $\tilde{X}$, given the original filtration. However, the process $\tilde{X}$ is never constructed, which obviously requires an appropriate randomization, and there is no mention of the induced filtration $\tilde{\mathcal{F}}$ and its relation to the original filtration $\mathcal{F}$. In fact, $\mathcal{F}$ enters only via the mentioned conditional probabilities.

For a semi-martingale $X$ with given local characteristics $\rho$, it is actually trivial to construct a process $\tilde{X}$ with conditionally independent increments, having the same local characteristics $\rho$. However, it is essential for our purposes that $X$ and $\tilde{X}$ should be tangential with respect to a common extended filtration $\tilde{\mathcal{F}}$, preserving all adaptedness and conditioning properties of $\mathcal{F}$, so that, in particular, every martingale or predictable process will remain a martingale or predictable process with respect to $\tilde{\mathcal{F}}$. This is why we require $\tilde{\mathcal{F}}$ to be a standard extension of $\mathcal{F}$. With this requirement, the construction becomes quite sophisticated and involves a subtle discussion of various properties of conditional independence, essentially contained in the proofs of Lemmas B.1, 3.2, and 3.4.

For clarity we begin with a preliminary result of independent interest. For any combination $\mathcal{X}$ of processes and filtrations, we define an $\mathcal{X}$-compensator as a compensator with respect to the filtration generated by $\mathcal{X}$. The definitions of $\mathcal{X}$-martingale, $\mathcal{X}$-adaptedness, etc., are similar.

LEMMA 3.1. Given a filtration $\mathcal{F}$ on $\mathrm{R}_{+}$, consider a continuous local martingale $M$ and an $S$-marked, adapted point process $\xi$ with compensator $\eta$. Form a Cox process $\zeta \Perp_{\eta} \mathcal{F}$ directed by $\eta$ and a Brownian motion $B \Perp(\zeta, \mathcal{F})$, put $N=$ $B \circ[M]$, and let $\mathcal{G}$ denote the filtration generated by $\mathcal{F}, \zeta$, and $N$. Then

(i) $\mathcal{G}$ is a standard extension of $\mathcal{F}$;

(ii) $N$ is a continuous local $\mathcal{G}$-martingale with $[N]=[M]$ a.s., and both $\xi$ and $\zeta$ have $\mathcal{G}$-compensator $\eta$;

(iii) when $\eta$ is continuous, it is also a $(\xi, \eta)$-compensator of $\xi$ and $a(\zeta, \eta)$ compensator of $\zeta$.

Pr o of. (i) Since $\zeta \Perp_{\eta} \mathcal{F}$ and $B \Perp_{(\zeta, \eta)} \mathcal{F}$, we have

$$
(\zeta, B) \Perp_{\eta} \mathcal{F}
$$


by the chain rule for conditional independence (cf. Proposition 6.8 in [12]), and so

$$
\left(\zeta^{t}, N^{t}\right) \underset{\eta,[M]}{\Perp} \mathcal{F}, \quad t \geqslant 0,
$$

where $\zeta^{t}$ denotes the restriction of $\zeta$ to $[0, t] \times S$. Using the conditioning criterion in Proposition 6.6 of [12] and the definitions of $\zeta$ and $N$, we further note that

$$
\left(\zeta^{t}, N^{t}\right) \underset{\eta^{t},[M]^{t}}{\Perp}(\eta,[M]), \quad t \geqslant 0 .
$$

Combining those relations and using the chain rule again, we obtain

$$
\left(\zeta^{t}, N^{t}\right) \underset{\eta^{t},[M]^{t}}{\Perp} \mathcal{F}, \quad t \geqslant 0
$$

which implies $\mathcal{G}_{t} \Perp_{\mathcal{F}_{t}} \mathcal{F}$ for all $t \geqslant 0$.

(ii) Since $B \Perp(\zeta, \mathcal{F})$, we get

$$
B \underset{[M], N^{s}}{\bigsqcup^{s}}\left(\mathcal{F}, \zeta, N^{s}\right), \quad s \geqslant 0
$$

and so

$$
\theta_{s} N \underset{[M], N^{s}}{\Perp} \mathcal{G}_{s}, \quad s \geqslant 0 .
$$

Combining with the relation $\theta_{s} N \Perp_{[M]} N^{s}$ and using the chain rule again, we obtain

$$
\theta_{s} N \frac{\Perp}{[M]} \mathcal{G}_{s}, \quad s \geqslant 0 .
$$

Localizing if necessary to ensure integrability, we get for any $s \geqslant t$ the desired martingale property

$$
\begin{aligned}
E\left(N_{t}-N_{s} \mid \mathcal{G}_{s}\right) & =E\left[E\left(N_{t}-N_{s} \mid \mathcal{G}_{s},[M]\right) \mid \mathcal{G}_{s}\right] \\
& =E\left[E\left(N_{t}-N_{s} \mid[M]\right) \mid \mathcal{G}_{s}\right]=0,
\end{aligned}
$$

and the associated rate property

$$
\begin{aligned}
E\left(N_{t}^{2}-N_{s}^{2} \mid \mathcal{G}_{s}\right) & =E\left[E\left(N_{t}^{2}-N_{s}^{2} \mid \mathcal{G}_{s},[M]\right) \mid \mathcal{G}_{s}\right] \\
& =E\left[E\left(N_{t}^{2}-N_{s}^{2} \mid[M]\right) \mid \mathcal{G}_{s}\right] \\
& =E\left[E\left([M]_{t}-[M]_{s} \mid[M]\right) \mid \mathcal{G}_{s}\right]
\end{aligned}
$$

proving the assertions for $N$.

Property (i) shows that $\eta$ remains a $\mathcal{G}$-compensator of $\xi$. Next, the relation $\zeta \Perp_{\eta} \mathcal{F}$ implies $\theta_{t} \zeta \Perp_{\left(\eta, \zeta^{t}\right)} \mathcal{F}_{t}$. Combining this with the Cox property $\theta_{t} \zeta \Perp_{\eta} \zeta^{t}$ 
and using the chain rule, we get $\theta_{t} \zeta \Perp_{\eta}\left(\zeta^{t}, \mathcal{F}_{t}\right)$. Invoking the tower property of conditional expectations and the Cox property of $\zeta$, we obtain

$$
\begin{aligned}
E\left(\theta_{t} \zeta \mid \mathcal{G}_{t}\right) & =E\left(\theta_{t} \zeta \mid \zeta^{t}, \mathcal{F}_{t}\right) \\
& =E\left[E\left(\theta_{t} \zeta \mid \zeta^{t}, \eta, \mathcal{F}_{t}\right) \mid \zeta^{t}, \mathcal{F}_{t}\right] \\
& =E\left[E\left(\theta_{t} \zeta \mid \eta\right) \mid \zeta^{t}, \mathcal{F}_{t}\right] \\
& =E\left(\theta_{t} \eta \mid \zeta^{t}, \mathcal{F}_{t}\right)=E\left(\theta_{t} \eta \mid \mathcal{G}_{t}\right)
\end{aligned}
$$

Since $\eta$ remains $\mathcal{G}$-predictable, it is then a $\mathcal{G}$-compensator of $\zeta$.

(iii) The martingale properties in (ii) extend to the filtrations generated by $(\xi, \eta)$ and $(\zeta, \eta)$, respectively, by the tower property of conditional expectations. Since $\eta$ is continuous and adapted to both filtrations, it is both $(\xi, \eta)$-predictable and $(\zeta, \eta)$-predictable. The assertions follow by combination of the mentioned properties.

Using the previous ideas, we may construct tangential processes with conditionally independent increments.

THEOREM 3.1. For any $\mathcal{F}$-semi-martingale $X$ with local characteristics $Y$, there exist a standard extension $\mathcal{G}$ of $\mathcal{F}$ and a $\mathcal{G}$-tangential semi-martingale $\tilde{X} \Perp_{Y} \mathcal{F}$, such that $\tilde{X}$ has conditionally independent increments given $Y$.

Pr o o f. Define the jump point process $\xi$ of $X$ as in (R.I), and let $\eta$ denote the $\mathcal{F}$-compensator of $\xi$. Further, let $M$ be the continuous martingale component of $X$, and let $A$ be the predictable drift component of $X$ with respect to an arbitrarily fixed truncation function. When $\eta$ is continuous, we may define $\zeta, N$, and $\mathcal{G}$ as in Lemma B.1, and construct an associated semi-martingale $\tilde{X}$ by compensating the jumps given by $\zeta$. Then $\tilde{X}$ has the same local characteristics $Y=([M], \eta, A)$ as $X$, so the two processes are $\mathcal{G}$-tangential. Since $\zeta \Perp_{\eta} \mathcal{F}$ and $B \Perp(\zeta, \mathcal{F})$, we have $\tilde{X} \Perp_{Y} \mathcal{F}$, and the independence properties of $B$ and $\zeta$ show that $\tilde{X}$ has conditionally independent increments.

The tangential property may fail when $\eta$ has discontinuities, since the projection $\bar{\zeta}=\zeta(\cdot \times S)$ may then have multiplicities, so that $\zeta$ is no longer a marked point process. We then need to replace the Cox process in Lemma 3.1 by a more general $S$-marked point process $\zeta$ on $\mathrm{R}_{+}$with conditionally independent increments given $\eta$, such that $E(\zeta \mid \eta)=\eta$ a.s. Note that the compensator $\eta$ of $\xi$ a.s. satisfies $\eta(\{t\} \times S) \leqslant 1$ for all $t \geqslant 0$, since the discontinuity set is covered by countably many predictable times.

Just as in the construction of Cox processes, it is enough to consider a nonrandom measure $\mu=\eta$ with this property, and construct an $S$-marked point process $\zeta$ with independent increments and intensity $\mu$. Then write as in ([2.3]

$$
\mu=\mu^{c}+\sum_{k \geqslant 1}\left(\delta_{t_{k}} \otimes \nu_{k}\right)
$$


where $\mu^{c}$ is continuous, the times $t_{k} \in(0, \infty)$ are distinct and at most countably many, and the $\nu_{k}$ are measures on $S$ with $\left\|\nu_{k}\right\| \leqslant 1$. Let $\alpha$ be a Poisson process on $\mathrm{R}_{+} \times S$ with intensity $\mu^{c}$, and choose some independent random elements $\beta_{k}$ in $S^{\Delta}$ with distributions $\nu_{k}$ on $S$. As in case of (2.2), the $S$-marked point process

$$
\zeta=\alpha+\sum_{k \geqslant 1}\left(\delta_{t_{k}} \otimes \delta_{\beta_{k}}\right)
$$

has clearly the desired properties. It is easy to check that the associated kernel is measurable in $\mu$, which allows us to construct an associated $S$-marked point process with conditionally independent increments and conditional intensity $\eta$. The assertions of Lemma 3.11 remain valid in this case, and the associated semi-martingale $\tilde{X}$ has local characteristics $Y$, so that $X$ and $\tilde{X}$ are indeed $\mathcal{G}$-tangential.

Given an $S$-marked point process $\xi$ on $(0, \infty)$, we may construct a symmetrization $\tilde{\xi}$ by attaching independent random signs to the points of $\xi$. We need to know how that affects the compensator. Since $\tilde{\xi}$ is not adapted, we must first extend the underlying filtration $\mathcal{F}$. Here we consider the more general case where $\tilde{\xi}$ is a $\nu$-randomization of $\xi$, for some probability kernel $\nu$ from $\mathrm{R}_{+} \times S$ to $T$, so that a unit mass at $(r, s) \in \mathrm{R}_{+} \times S$ is equipped with a mark in $T$ with distribution $\nu_{r, s}$, independently for all points of $\xi$.

LEMma 3.2. Given an $\mathcal{F}$-adapted point process $\xi$ on $(0, \infty) \times S$ with compensator $\hat{\xi}$ and a probability kernel $\nu$ from $\mathrm{R}_{+} \times S$ to $T$, let $\eta$ be a $\nu$-randomization of $\xi$ on $\mathrm{R}_{+} \times S \times T$, and write $\mathcal{G}$ for the right-continuous filtration generated by $\mathcal{F}$ and $\eta$. Then $\mathcal{G}$ is a standard extension of $\mathcal{F}$, and $\eta$ has $\mathcal{G}$-compensator $\hat{\eta}=\hat{\xi} \otimes \nu$.

Proof. For any $t \geqslant 0$, let $\xi_{t}$ and $\eta_{t}$ denote the restrictions of $\xi$ and $\eta$ to $[0, t] \times S$ and $[0, t] \times S \times T$, respectively, and put $\eta_{t}^{\prime}=\eta-\eta_{t}$. Then clearly

$$
\eta \Perp_{\xi} \mathcal{F}, \quad \eta_{t} \Perp_{\xi} \eta_{t}^{\prime}, \quad \eta_{t} \Perp_{\xi_{t}} \xi
$$

Using the first relation, combining with the other two, and invoking the chain rule for conditional independence (cf. Proposition 6.8 in [12]), we obtain

$$
\eta_{t} \Perp_{\xi, \eta_{t}^{\prime}} \mathcal{F}, \quad \eta_{t} \Perp_{\xi_{t}}\left(\eta_{t}^{\prime}, \mathcal{F}\right),
$$

and so

$$
\eta_{t} \Perp_{\mathcal{F}_{t}}\left(\eta_{t}^{\prime}, \mathcal{F}\right), \quad\left(\eta_{t}, \mathcal{F}_{t}\right) \Perp_{\mathcal{F}_{t}}\left(\eta_{t}^{\prime}, \mathcal{F}\right)
$$

In the last relation we may approximate from the right to get $\mathcal{G}_{t} \Perp_{\mathcal{F}_{t}} \mathcal{F}$, which shows that $\mathcal{G}$ is a standard extension of $\mathcal{F}$. 
Using the latter property, the chain rule for conditional expectations, the relation $\eta \Perp_{\xi} \mathcal{F}$, Fubini's theorem, and the definitions of randomization and compensation, we get on $(t, \infty) \times S \times T$ for arbitrary $t \geqslant 0$

$$
\begin{aligned}
E\left(\eta \mid \mathcal{G}_{t}\right) & =E\left(\eta \mid \mathcal{F}_{t}\right)=E\left[E\left(\eta \mid \mathcal{F}_{t}, \xi\right) \mid \mathcal{F}_{t}\right] \\
& =E\left[E(\eta \mid \xi) \mid \mathcal{F}_{t}\right]=E\left(\xi \otimes \nu \mid \mathcal{F}_{t}\right) \\
& =E\left(\xi \mid \mathcal{F}_{t}\right) \otimes \nu=E\left(\hat{\xi} \mid \mathcal{F}_{t}\right) \otimes \nu \\
& =E\left(\hat{\xi} \otimes \nu \mid \mathcal{F}_{t}\right)=E\left(\hat{\xi} \otimes \nu \mid \mathcal{G}_{t}\right) .
\end{aligned}
$$

Here, e.g., $E\left(\eta \mid \mathcal{F}_{t}\right)$ denotes the a.s. unique $\mathcal{F}_{t}$-measurable random measure such that $E\left(\eta \mid \mathcal{F}_{t}\right) f=E\left(\eta f \mid \mathcal{F}_{t}\right)$ a.s. for any measurable function $f \geqslant 0$. Since $\hat{\eta}=$ $\hat{\xi} \otimes \nu$ is $\mathcal{F}$-predictable and hence even $\mathcal{G}$-predictable, we conclude that $\hat{\eta}$ is indeed a $\mathcal{G}$-compensator of $\eta$.

The last result has a partial converse, given here under some simplifying assumptions.

LEMMA 3.3. Let $\xi$ be an $\mathcal{F}$-adapted point process on $(0, \infty)$ with marks in $S \times T$, such that $\bar{\xi}=\xi(\cdot \times S \times T)$ is a.s. locally finite and unbounded, and suppose that $\xi$ has compensator $\hat{\xi}=\eta \otimes \nu$ for a predictable random measure $\eta$ on $\mathrm{R}_{+} \times S$ and a non-random probability measure $\nu$ on $T$. Then $\zeta=\xi(\cdot \times T)$ is an $S$-marked point process on $(0, \infty)$ with compensator $\eta$, and the associated sequence $\gamma=\left(\gamma_{k}\right)$ of T-marks is i.i.d. $\nu$. However, $\zeta$ and $\gamma$ need not be independent.

Pro of. The assertion for $\zeta$ being obvious by projection, we turn to the one for $\gamma$. Letting $\bar{\xi}$ have supporting times $\tau_{1}<\tau_{2}<\ldots$ and putting $\tau_{0}=0$, we introduce the discrete filtration $\mathcal{G}_{k}=\mathcal{F}_{\tau_{k}}, k \in \mathrm{Z}_{+}$. Then $\gamma$ is clearly $\mathcal{G}$-adapted, and since $\bar{\xi}$ has exactly one point in each interval $\left(\tau_{k-1}, \tau_{k}\right]$, we see by optional sampling that $\gamma$ has $\mathcal{G}$-compensator

$$
\begin{aligned}
\hat{\gamma}_{k} & =E\left[\eta\left(\left(\tau_{k-1}, \tau_{k}\right] \times S\right) \mid \mathcal{G}_{k-1}\right] \nu \\
& =E\left[\zeta\left(\left(\tau_{k-1}, \tau_{k}\right] \times S\right) \mid \mathcal{G}_{k-1}\right] \nu=\nu
\end{aligned}
$$

a.s. for each $k \in \mathrm{N}$. It remains to apply the discrete-time version of Jacod's theorem.

To prove the last assertion, let $\xi$ be a unit rate Poisson process on $\mathrm{R}_{+}$, and attach some i.i.d. $U(0,1)$ marks $\gamma_{1}, \gamma_{2}, \ldots$ to the points $\tau_{1}, \tau_{2}, \ldots$ of $\xi$. Then form a new process $\xi^{\prime}$ by deleting all points of $\xi$ in the interval $\left(\tau_{1}, \tau_{1}+\gamma_{1}\right]$. The remaining marks $\gamma_{k}^{\prime}$ are again i.i.d. $\nu$, e.g., by optional skipping (cf. Theorem 11.13 in [12]), but they are not independent of $\xi^{\prime}$, since the latter process depends on the first mark $\gamma_{1}$.

The following preservation property is needed in the next section. 
LEMMA 3.4. Given an $\mathcal{F}$-semi-martingale $X$ with local characteristics $\rho$, let $Y$ and $Y^{\prime}$ be $\mathcal{G}$-tangential to $X$ with conditionally independent increments, where $\mathcal{G}$ is a standard extension of $\mathcal{F}$, and suppose that $\mathcal{F}, Y$, and $Y^{\prime}$ are conditionally independent given $\rho$. Then $X-Y$ and $Y-Y^{\prime}$ are $\mathcal{G}$-tangential.

Proof. The only difficulty is to verify the tangential property for the associated jump point processes. Then let $\xi, \eta$, and $\eta^{\prime}$ be the jump processes of $X, Y$, and $Y^{\prime}$, and let $\hat{\xi}$ denote the associated compensator, so that $\mathcal{F}, \eta$, and $\eta^{\prime}$ are conditionally independent given $\hat{\xi}$. On the continuity set $A$ of $\hat{\xi}$, the processes $\eta$ and $\eta^{\prime}$ are even conditionally Poisson $\hat{\xi}$, and so the discontinuity sets of the three processes are a.s. disjoint on $A$, and the compensators of the jump processes of $X-Y$ and $Y-Y^{\prime}$ agree a.s. on $A$.

Since $\hat{\xi}$ is predictable, its discontinuity set $A^{c}$ is covered by the graphs of countably many predictable times $\tau_{1}, \tau_{2}, \ldots$ (cf. Proposition 25.17 in [12]), and so it suffices to show that the two compensators agree a.s. at any predictable time $\tau<\infty$. Let $\tau$ be announced by the optional times $\sigma_{1}, \sigma_{2}, \ldots$. Since $1_{B} 1_{[\tau]}$ is predictable for any $B \in \mathcal{G}_{\sigma_{n}}$, we note that $E\left(\Delta \hat{\xi}_{\tau} \mid \mathcal{G}_{\sigma_{n}}\right)=E\left(\Delta \xi_{\tau} \mid \mathcal{G}_{\sigma_{n}}\right)$, which extends to $E\left(\Delta \hat{\xi}_{\tau} \mid \mathcal{G}_{\tau-}\right)=E\left(\Delta \xi_{\tau} \mid \mathcal{G}_{\tau-}\right)$ by martingale convergence (cf. Lemma 25.2 and Theorem 7.23 in [12]). Since $\Delta \hat{\xi}_{\tau}$ is $\mathcal{G}_{\tau-}$-measurable by Lemma 25.3 in [12], we obtain a.s.

$$
\Delta \hat{\xi}_{\tau}=E\left(\Delta \xi_{\tau} \mid \mathcal{G}_{\tau-}\right)=E\left(\Delta \eta_{\tau} \mid \mathcal{G}_{\tau-}\right)=E\left(\Delta \eta_{\tau}^{\prime} \mid \mathcal{G}_{\tau-}\right) .
$$

Noting that $\eta$ has compensator $\hat{\xi}$ with respect to the extended filtration $\mathcal{H}_{t}=$ $\mathcal{G}_{t} \vee \mathcal{F}_{\infty}$, we obtain $E \eta V=E \hat{\xi} V$ for any $\mathcal{H}$-predictable process $V \geqslant 0$. Given a predictable time $\tau$ as above, we may choose $V=1_{B} 1_{[\tau]}$ for any $B \in \mathcal{H}_{\tau-}$ to get a.s.

$$
E\left(\Delta \eta_{\tau} \mid \mathcal{H}_{\tau-}\right)=E\left(\Delta \hat{\xi}_{\tau} \mid \mathcal{H}_{\tau-}\right)=\Delta \hat{\xi}_{\tau}
$$

where the last equality holds since $\Delta \hat{\xi}_{\tau}$ is $\mathcal{G}_{\tau-}$-measurable. Since the same relations hold for the original filtration $\mathcal{G}$, we conclude that a.s.

$$
E\left(\Delta \eta_{\tau} \mid \mathcal{G}_{\tau-}, \mathcal{F}_{\infty}\right)=E\left(\Delta \eta_{\tau} \mid \mathcal{G}_{\tau-}\right),
$$

which means that $\Delta \eta_{\tau}$ and $\mathcal{F}_{\infty}$ are conditionally independent given $\mathcal{G}_{\tau-}$. By a similar argument applied to $\Delta \eta_{\tau}^{\prime}$, we obtain

$$
\Delta \xi_{\tau} \frac{\Perp}{\mathcal{G}_{\tau-}} \Delta \eta_{\tau} \frac{\Perp}{\mathcal{G}_{\tau-}} \Delta \eta_{\tau}^{\prime} .
$$

Combining with (3.1), we get a.s.

$$
E\left[\Delta\left(\xi_{\tau}, \eta_{\tau}\right) \mid \mathcal{G}_{\tau-}\right]=E\left[\Delta\left(\eta_{\tau}, \eta_{\tau}^{\prime}\right) \mid \mathcal{G}_{\tau-}\right],
$$

and it follows easily that the compensators of the jump processes of $X-Y$ and $Y-Y^{\prime}$ agree a.s. at $\tau$. 
We conclude with a simple technical fact, needed in Section 6.

LEMMA 3.5. Let $\xi$ be an $\mathcal{F}$-adapted, $T$-marked point process on $(0, \infty)$ with $\mathcal{F}$-compensator $\eta$. Then $\eta$ remains an $\mathcal{F}$-compensator of $\xi$, conditionally on $\mathcal{F}_{0}$.

Proof. It is enough to verify that $E\left(\xi V \mid \mathcal{F}_{0}\right)=E\left(\eta V \mid \mathcal{F}_{0}\right)$ a.s. for any $\mathcal{F}$ predictable process $V \geqslant 0$, which holds since the process $1_{A} V$ is again $\mathcal{F}$-predictable for any $A \in \mathcal{F}_{0}$.

\section{TANGENTIAL COMPARISON}

Here we prove the basic comparison theorems for tangential processes, extending the discrete-time results of Zinn [27], Hitchenko [3], and Kwapień and Woyczyński [2]]. We also prove some related separation properties, and show how the results lead to some general equivalence statements for broad classes of tangential or semi-tangential processes. Say that a semi-martingale $X$ is conditionally symmetric if $X$ and $-X$ are tangential.

A non-decreasing, continuous function $\varphi$ on $\mathrm{R}_{+}$with $\varphi(0)=0$ is said to have moderate growth if $\varphi(2 x) \leqslant c \varphi(x), x>0$, for some constant $c>0$. Then there exists a function $h>0$ on $(0, \infty)$ such that $\varphi(c x) \leqslant h(c) \varphi(x)$ for all $c, x>0$. The stated condition holds, in particular, for all power functions $\varphi(x)=|x|^{p}$ with $p>0$, as well as for $\varphi(x)=x \wedge 1$ and $\varphi(x)=1-e^{-x}$. Note that the composition of two functions of moderate growth has the same property.

THEOREM 4.1. Let the processes $X$ and $Y$ be tangential, increasing or conditionally symmetric. Then for any non-decreasing, continuous function of moderate growth $\varphi$ on $\mathrm{R}_{+}$with $\varphi(0)=0$, we have

$$
E \varphi\left(X^{*}\right) \asymp E \varphi\left(Y^{*}\right)
$$

where the domination constants depend only on $\varphi$. When $\varphi$ is convex, this remains true for any weakly tangential local martingales $X$ and $Y$.

Our proof is a continuous-time version of the argument in [21]. We begin with some tail estimates of independent interest.

LEMMA 4.1. Let the processes $X$ and $Y$ be tangential, increasing or conditionally symmetric. Then for any $c, x>0$,

(i) $P\left\{(\Delta X)^{*}>x\right\} \leqslant 2 P\left\{(\Delta Y)^{*}>x\right\}$;

(ii) $P\left\{X^{*}>x\right\} \leqslant 3 P\left\{Y^{*}>c x\right\}+4 c$.

Pro of. (i) Let $\xi$ and $\eta$ be the jump point processes of $X$ and $Y$. Fix any $x>0$, and introduce the optional time

$$
\tau=\inf \left\{t>0 ;\left|\Delta Y_{t}\right|>x\right\} .
$$


Since the set $(0, \tau]$ is predictable by Lemma 25.1 in [12], we get

$$
\begin{aligned}
P\left\{(\Delta X)^{*}>x\right\} & \leqslant P\{\tau<\infty\}+E \xi\left((0, \tau] \times[-x, x]^{c}\right) \\
& \leqslant P\{\tau<\infty\}+E \eta\left((0, \tau] \times[-x, x]^{c}\right) \\
& =2 P\{\tau<\infty\}=2 P\left\{(\Delta Y)^{*}>x\right\} .
\end{aligned}
$$

(ii) Fix any $c, x>0$. When $X$ and $Y$ are increasing, we form $\hat{X}$ and $\hat{Y}$ by omitting all jumps greater than $2 c x$, which clearly preserves the tangential relation. If $X$ and $Y$ are instead conditionally symmetric, we form $\hat{X}$ and $\hat{Y}$ by omitting all jumps of modulus greater than $2 c x$. The symmetry implies that $\hat{X}$ and $\hat{Y}$ are local $L^{2}$-martingales with jumps a.s. bounded by $4 c x$. They also remain tangential, and the tangential relation carries over to the quadratic variation processes $[\hat{X}]$ and $[\hat{Y}]$.

Now introduce the optional time

$$
\tau=\inf \left\{t>0 ;\left|\hat{Y}_{t}\right|>c x\right\} .
$$

For increasing $X$ and $Y$, the tangential property of $(\hat{X}, \hat{Y})$ yields

$$
\begin{aligned}
x P\left\{\hat{X}_{\tau}>x\right\} & \leqslant E \hat{X}_{\tau}=E \hat{Y}_{\tau} \\
& =E \hat{Y}_{\tau-}+E \Delta \hat{Y}_{\tau} \leqslant 3 c x .
\end{aligned}
$$

If instead $X$ and $Y$ are conditionally symmetric, we may use the Bernstein-Lévy and Jensen inequalities (cf. Proposition 7.15 and Lemma 3.5 in [12]), integration by parts (cf. Theorem 26.6 (vii) in [12]), and the tangential properties and bounds to get

$$
\begin{aligned}
\left(x P\left\{\hat{X}_{\tau}^{*}>x\right\}\right)^{2} & \leqslant\left(E\left|\hat{X}_{\tau}\right|\right)^{2} \leqslant E \hat{X}_{\tau}^{2} \\
& =E[\hat{X}]_{\tau}=E[\hat{Y}]_{\tau}=E \hat{Y}_{\tau}^{2} \\
& \leqslant E\left(\left|\hat{Y}_{\tau-}\right|+\left|\Delta \hat{Y}_{\tau}\right|\right)^{2} \leqslant(4 c x)^{2} .
\end{aligned}
$$

Thus, in both cases $P\left\{\hat{X}_{\tau}^{*}>x\right\} \leqslant 4 c$.

By the definitions of $\hat{X}$ and $\hat{Y}$,

$$
\begin{aligned}
& \left\{(\Delta X)^{*} \leqslant 2 c x\right\}=\{X=\hat{X}\}, \\
& \left\{(\Delta Y)^{*} \leqslant 2 c x\right\}=\{Y=\hat{Y}\} .
\end{aligned}
$$

Using the second relation and noting that $Y^{*} \geqslant \frac{1}{2}(\Delta Y)^{*}$, we get

$$
\begin{aligned}
\{\tau<\infty\} & =\left\{\hat{Y}^{*}>c x\right\} \\
& \subset\left\{\hat{Y}^{*}>c x, Y=\hat{Y}\right\} \cup\left\{(\Delta Y)^{*}>2 c x\right\} \\
& \subset\left\{Y^{*}>c x\right\} .
\end{aligned}
$$


Combining with the previous tail estimate along with (4.1) and (i), we obtain

$$
\begin{aligned}
P\left\{X^{*}>x\right\} & \leqslant P\left\{(\Delta X)^{*}>2 c x\right\}+P\left\{\hat{X}^{*}>x\right\} \\
& \leqslant 2 P\left\{(\Delta Y)^{*}>2 c x\right\}+P\{\tau<\infty\}+P\left\{\hat{X}_{\tau}^{*}>x\right\} \\
& \leqslant 3 P\left\{Y^{*}>c x\right\}+4 c .
\end{aligned}
$$

Proof of Theorem 4. 1 . For any $c, x>0$, we introduce the optional times

$$
\begin{aligned}
\tau & =\inf \left\{t>0 ;\left|X_{t}\right|>x\right\}, \\
\sigma & =\inf \left\{t>0 ; P\left[\left(\theta_{t} Y\right)^{*}>c x \mid \mathcal{F}_{t}\right]>c\right\} .
\end{aligned}
$$

Since $\theta_{\tau} X$ and $\theta_{\tau} Y$ remain conditionally tangential given $\mathcal{F}_{\tau}$, Lemma 4.1 (ii) yields a.s. on $\{\tau<\sigma\}$

$$
P\left[\left(\theta_{\tau} X\right)^{*}>x \mid \mathcal{F}_{\tau}\right] \leqslant 3 P\left[\left(\theta_{\tau} Y\right)^{*}>c x \mid \mathcal{F}_{\tau}\right]+4 c \leqslant 7 c,
$$

and since $\{\tau<\sigma\} \in \mathcal{F}_{\tau}$ by Lemma 25.2 in [12], we get

$$
\begin{aligned}
P\left\{X^{*}>3 x,(\Delta X)^{*} \leqslant x, \sigma=\infty\right\} & \leqslant P\left\{\left(\theta_{\tau} X\right)^{*}>x, \tau<\sigma\right\} \\
& =E\left[P\left[\left(\theta_{\tau} X\right)^{*}>x \mid \mathcal{F}_{\tau}\right] ; \tau<\sigma\right] \\
& \leqslant 7 c P\{\tau<\infty\}=7 c P\left\{X^{*}>x\right\} .
\end{aligned}
$$

Furthermore, by Lemma 4.1 (i),

$$
P\left\{(\Delta X)^{*}>x\right\} \leqslant 2 P\left\{(\Delta Y)^{*}>x\right\} \leqslant 2 P\left\{Y^{*}>x / 2\right\},
$$

and the Bernstein-Lévy inequality (Proposition 7.15 in [12]) yields

$$
\begin{aligned}
P\{\sigma<\infty\} & =P\left\{\sup _{t} P\left[\left(\theta_{t} Y\right)^{*}>c x \mid \mathcal{F}_{t}\right]>c\right\} \\
& \leqslant P\left\{\sup _{t} P\left[Y^{*}>c x / 2 \mid \mathcal{F}_{t}\right]>c\right\} \\
& \leqslant c^{-1} P\left\{Y^{*}>c x / 2\right\} .
\end{aligned}
$$

Combining the last three estimates, we obtain

$$
\begin{aligned}
& P\left\{X^{*}>3 x\right\} \\
& \leqslant P\left\{X^{*}>3 x,(\Delta X)^{*} \leqslant x, \sigma=\infty\right\}+P\left\{(\Delta X)^{*}>x\right\}+P\{\sigma<\infty\} \\
& \leqslant 7 c P\left\{X^{*}>x\right\}+2 P\left\{Y^{*}>x / 2\right\}+c^{-1} P\left\{Y^{*}>c x / 2\right\} .
\end{aligned}
$$

Since $\varphi$ is non-decreasing of moderate growth, we have $\varphi(r x) \leqslant h(r) \varphi(x)$ for some function $h>0$, and so

$$
\begin{aligned}
\left(h_{3}^{-1}-7 c\right) E \varphi\left(X^{*}\right) & \leqslant E \varphi\left(X^{*} / 3\right)-7 c E \varphi\left(X^{*}\right) \\
& \leqslant 2 E \varphi\left(2 Y^{*}\right)+c^{-1} E \varphi\left(2 Y^{*} / c\right) \\
& \leqslant\left(2 h_{2}+c^{-1} h_{2 / c}\right) E \varphi\left(Y^{*}\right) .
\end{aligned}
$$

We may finally choose $c<\left(7 h_{3}\right)^{-1}$ to get $E \varphi\left(X^{*}\right) \leq E \varphi\left(Y^{*}\right)$. 
Now let $\varphi$ be a convex function of moderate growth, and note that the function $\varphi\left(x^{1 / 2}\right)$ is again of moderate growth. For any weakly tangential local martingales $X$ and $Y$, the processes $[X]$ and $[Y]$ are non-decreasing and strictly tangential, and so by the previous result and a version of the BDG-inequalities (cf. [1]]), we obtain

$$
E \varphi\left(X^{*}\right) \asymp E \varphi\left([X]_{\infty}^{1 / 2}\right) \asymp E \varphi\left([Y]_{\infty}^{1 / 2}\right) \asymp E \varphi\left(Y^{*}\right),
$$

which proves the last assertion.

The conditional symmetry of $X$ and $Y$ seems to be needed only for the proof of Lemma 1.] (ii), which uses the fact that the jumps of size greater than $2 c x$ are self-compensating, in the sense that the truncated processes $\hat{X}$ and $\hat{Y}$ are again local martingales for arbitrary $c$ and $x$. Otherwise, we would need to subtract the compensated jumps, which would violate the set inclusions (4. (1). In general, it is hard to think of a truncation (even in discrete time, cf. [21], pp. 115ff.) that would both preserve the martingale property and satisfy (4.J). Yet one might hope that the last theorem would remain true under more general conditions.

We continue with a one-sided comparison, which extends the discrete-time version in [21], p. 134.

THEOREM 4.2. Let us consider some tangential semi-martingales $X$ and $Y$, where $Y$ has conditionally independent increments. Then for any continuous, nondecreasing function $\varphi$ of moderate growth, we have

$$
E \varphi\left(X^{*}\right) \leq E \varphi\left(Y^{*}\right),
$$

where the domination constant depends only on $\varphi$.

Proof. Let $\rho$ denote the local characteristics of $X$. Proceeding as in Theorem B.], we may construct two tangential processes $Y^{\prime}$ and $Y^{\prime \prime}$ with conditionally independent increments, such that $Y^{\prime}, Y^{\prime \prime}$, and $\mathcal{F}$ are conditionally independent given $\rho$. Since $Y^{\prime} \stackrel{d}{=} Y^{\prime \prime} \stackrel{d}{=} Y$ and the processes $X-Y^{\prime}$ and $Y^{\prime}-Y^{\prime \prime}$ are conditionally symmetric and tangential by Lemma 3.4, we see from the growth property of $\varphi$ and Theorem 4 . 1 that

$$
\begin{aligned}
E \varphi\left(X^{*}\right) & \leq E \varphi \circ\left(X-Y^{\prime}\right)^{*}+E \varphi\left(Y^{\prime *}\right) \\
& \leq E \varphi \circ\left(Y^{\prime}-Y^{\prime \prime}\right)^{*}+E \varphi\left(Y^{\prime *}\right) \\
& \leq 2 E \varphi\left(Y^{\prime *}\right)+E \varphi\left(Y^{\prime \prime}\right) \\
& =3 E \varphi\left(Y^{*}\right),
\end{aligned}
$$

where the domination constants depend only on $\varphi$.

The previous results yield some useful comparisons of boundedness or convergence for suitable pairs of strictly or weakly tangential processes. We begin with the following equivalences. 
THEOREM 4.3. Let $(X, Y)$ and $\left(X_{n}, Y_{n}\right), n \in \mathrm{N}$, be pairwise tangential, increasing or conditionally symmetric processes, or weakly tangential local martingales with uniformly bounded jumps. Then

(i) $\left\{X^{*}<\infty\right\}=\left\{Y^{*}<\infty\right\}$ a.s.;

(ii) $X_{n}^{*} \stackrel{P}{\rightarrow} 0 \Leftrightarrow Y_{n}^{*} \stackrel{P}{\rightarrow} 0$.

Note that (i) is stronger than the equivalence

$$
X^{*}<\infty \text { a.s. } \Leftrightarrow Y^{*}<\infty \text { a.s., }
$$

which follows from (ii) with $X_{n}=X / n$ and $Y_{n}=Y / n$. Applying (ii) to the processes $X_{n}=\theta_{n} X-X(n)$ and $Y_{n}=\theta_{n} Y-Y(n)$, we note that also

$$
X \text { converges a.s. } \Leftrightarrow Y \text { converges a.s. }
$$

Pro of. (i) First let $X$ and $Y$ be tangential, increasing or conditionally symmetric. Fixing any $n \in \mathrm{N}$, we consider the optional time

$$
\tau=\inf \left\{t>0 ;\left|X_{t}\right|>n\right\} .
$$

For any $a>0$, we may apply Theorem 4.1 to the tangential, increasing or conditionally symmetric processes $a X^{\tau}$ and $a Y^{\tau}$ and the function $\varphi(x)=x \wedge 1$ with associated domination constant $c>0$ to get

$$
\begin{aligned}
P\left\{X^{*} \leqslant n, Y^{*}=\infty\right\} & =P\left\{\tau=\infty, Y_{\tau}^{*}=\infty\right\} \\
& \leqslant P\left\{Y_{\tau}^{*}=\infty\right\} \leqslant E\left(a Y_{\tau}^{*} \wedge 1\right) \\
& \leqslant c E\left(a X_{\tau}^{*} \wedge 1\right) .
\end{aligned}
$$

Since $X_{\tau}^{*} \leqslant n+\left|\Delta X_{\tau}\right|<\infty$ a.s., the right-hand side tends to zero as $a \rightarrow 0$ by dominated convergence, and so

$$
P\left\{X^{*}<\infty, Y^{*}=\infty\right\} \leqslant \sum_{n \geqslant 1} P\left\{X^{*} \leqslant n, Y^{*}=\infty\right\}=0,
$$

which means that $Y^{*}<\infty$ a.s. on $\left\{X^{*}<\infty\right\}$. By symmetry we may interchange the roles of $X$ and $Y$.

Next let $X$ and $Y$ be weakly tangential local martingales with jumps bounded by $b$. For $\tau$ as above, the last statement of Theorem 4.1 yields $E X_{\tau}^{*} \asymp E Y_{\tau}^{*}$, and so for any $a>0$ we get as before

$$
\begin{aligned}
P\left\{X^{*} \leqslant n, Y^{*}=\infty\right\} & =P\left\{\tau=\infty, Y_{\tau}^{*}=\infty\right\} \\
& \leqslant P\left\{Y_{\tau}^{*}=\infty\right\} \leqslant a E Y_{\tau}^{*} \\
& \leqslant a c E X_{\tau}^{*}
\end{aligned}
$$


for some constant $c>0$. Since $a>0$ was arbitrary and $X_{\tau}^{*} \leqslant n+b<\infty$, the probability on the left equals zero. The proof may now be completed as before.

(ii) The statement for tangential, increasing or conditionally symmetric processes is immediate from Theorem 4.1] with $\varphi(x)=x \wedge 1$. Now let $X_{n}$ and $Y_{n}$ be weakly tangential local martingales with jumps bounded by $b$. Suppose that $X_{n}^{*} \stackrel{P}{\rightarrow} 0$, and introduce the optional times

$$
\tau_{n}=\inf \left\{t>0 ;\left|X_{n}(t)\right|>1\right\}, \quad n \in \mathrm{N} .
$$

Since $X_{n}^{*}\left(\tau_{n}\right) \leqslant 1+b$, and $E X_{n}^{*}\left(\tau_{n}\right) \asymp E Y_{n}^{*}\left(\tau_{n}\right)$ by Theorem 4.1, we get

$$
\begin{aligned}
E\left[Y_{n}^{*} \wedge 1\right] & \leqslant P\left\{\tau_{n}<\infty\right\}+E Y_{n}^{*}\left(\tau_{n}\right) \\
& \leq P\left\{X_{n}^{*}>1\right\}+E X_{n}^{*}\left(\tau_{n}\right) \rightarrow 0,
\end{aligned}
$$

which shows that $Y_{n}^{*} \stackrel{P}{\rightarrow} 0$. By symmetry we may interchange the roles of $X$ and $Y$.

For any local martingale $X$ and constant $r>0$, we may write $X=X^{\prime}+X^{\prime \prime}$, where $X^{\prime}$ is the sum of all compensated jumps in $X$ of modulus greater than $r$. Recall from Lemma 26.5 in [12] that $X^{\prime}$ has locally integrable variation, whereas $X^{\prime \prime}$ is a local martingale with jumps bounded by $2 r$. The following preliminary result, of some independent interest, shows that we may sometimes separate the large and small jumps.

Lemma 4.2. Let $X$ and $X_{1}, X_{2}, \ldots$ be local martingales with conditionally symmetric or independent increments, and write $X=X^{\prime}+X^{\prime \prime}$ and $X_{n}=X_{n}^{\prime}+$ $X_{n}^{\prime \prime}$, where $X^{\prime}$ and $X_{n}^{\prime}$ are the sums of all compensated jumps in $X$ and $X_{n}$ of size greater than one. Then

(i) $\left\{X^{*}<\infty\right\}=\left\{X^{\prime *}+X^{\prime \prime *}<\infty\right\}$ a.s.;

(ii) $X_{n}^{*} \stackrel{P}{\rightarrow} 0 \Leftrightarrow X_{n}^{*}+X_{n}^{\prime \prime *} \stackrel{P}{\rightarrow} 0$.

Since trivially $X^{*} \leqslant X^{\prime *}+X^{\prime \prime *}$ and $X_{n}^{*} \leqslant X_{n}^{\prime *}+X_{n}^{\prime \prime *}$, the main contributions are the inclusion and implication to the right.

Pro of. (i) First let $X$ be a local martingale with conditionally independent increments. Conditioning on the local characteristics, we may reduce to the case where $X$ is centered with strictly independent increments. Since each side has then probability 0 or 1 by Kolmogorov's $0-1$ law, it suffices to show that $X^{*}<\infty$ a.s. implies $X^{\prime *}+X^{\prime \prime *}<\infty$ a.s. Thus, we need to show that $X^{*}<\infty$ a.s. implies $X^{\prime \prime *}<\infty$ a.s. Letting $\tilde{X}$ and $\tilde{X}^{\prime \prime}$ be symmetrizations of $X$ and $X^{\prime \prime}$, respectively, we note that $X^{*}<\infty$ a.s. implies $\tilde{X}^{*}<\infty$ a.s., and so $\tilde{X}^{\prime \prime *}<\infty$ a.s. by Lemma 2.2 (i). Since $X^{\prime \prime}$ has bounded jumps, Corollary [2.] (i) shows that even $X^{\prime \prime *}<\infty$ a.s. 
If $X$ is instead conditionally symmetric, then by Theorem B.] we may choose $Y$ to be tangential to $X$ with conditionally independent increments. Introducing the corresponding decomposition $Y=Y^{\prime}+Y^{\prime \prime}$, we note that $Y^{\prime}$ and $Y^{\prime \prime}$ are tangential to $X^{\prime}$ and $X^{\prime \prime}$, respectively. Hence, the tangential case of Theorem 4.3 (i) yields a.s. the equalities

$$
\begin{aligned}
\left\{X^{*}<\infty\right\} & =\left\{Y^{*}<\infty\right\}, \\
\left\{X^{\prime *}+X^{\prime \prime *}<\infty\right\} & =\left\{Y^{\prime *}+Y^{\prime \prime *}<\infty\right\},
\end{aligned}
$$

which reduces the proof to the previous case of processes with conditionally independent increments.

(ii) If the $X_{n}$ are conditionally symmetric, then by Theorem B.] we may choose $Y_{1}, Y_{2}, \ldots$ to be pairwise tangential to $X_{1}, X_{2}, \ldots$ with conditionally independent increments. Writing $Y_{n}=Y_{n}^{\prime}+Y_{n}^{\prime \prime}$ as before, we note that $Y_{n}^{\prime}$ and $Y_{n}^{\prime \prime}$ are tangential to $X_{n}^{\prime}$ and $X_{n}^{\prime \prime}$, respectively. The tangential case of Theorem 4.3 (ii) yields

$$
\begin{aligned}
X_{n}^{*} \stackrel{P}{\rightarrow} 0 & \Leftrightarrow \quad Y_{n}^{*} \stackrel{P}{\rightarrow} 0, \\
X_{n}^{\prime *}+X_{n}^{\prime \prime *} \stackrel{P}{\rightarrow} 0 & \Leftrightarrow \quad Y_{n}^{\prime *}+Y_{n}^{\prime \prime *} \stackrel{P}{\rightarrow} 0,
\end{aligned}
$$

which reduces the proof to the case of processes with conditionally independent increments. By the same argument, we may further assume that the processes $X_{n}$ are conditionally independent, given the local characteristics of the whole family $\left(X_{n}\right)$.

In the latter case, we may turn to subsequences to ensure a.s. convergence, and then condition on the set of local characteristics, to reduce to the case of processes with strictly independent increments. Introducing the symmetrizations $\tilde{X}_{n}$ and $\tilde{X}_{n}^{\prime \prime}$, we note as before that $X_{n}^{*} \stackrel{P}{\rightarrow} 0$ implies $\tilde{X}_{n}^{*} \stackrel{P}{\rightarrow} 0$, which yields $\tilde{X}_{n}^{\prime \prime *} \stackrel{P}{\rightarrow} 0$ by Lemma 2.2 (ii). Then $X_{n}^{\prime \prime *} \stackrel{P}{\rightarrow} 0$ by Corollary [2.] (ii), and so $X_{n}^{\prime *}+X_{n}^{\prime \prime *} \leqslant$ $X_{n}^{*}+2 X_{n}^{\prime \prime *} \stackrel{P}{\rightarrow} 0$.

Say that two local martingales $X$ and $Y$ are semi-tangential if there exists a constant $r>0$ with corresponding decompositions $X=X^{\prime}+X^{\prime \prime}$ and $Y=Y^{\prime}+$ $Y^{\prime \prime}$, such that $X^{\prime}$ and $Y^{\prime}$ are tangential while $X^{\prime \prime}$ and $Y^{\prime \prime}$ are weakly tangential. More generally, let us say that two sequences of local martingales $X_{1}, X_{2}, \ldots$ and $Y_{1}, Y_{2}, \ldots$ are pairwise uniformly semi-tangential if $X_{n}$ and $Y_{n}$ are semi-tangential for every $n \in \mathrm{N}$ with a truncation level $r>0$ independent of $n$.

Using the previous lemma, we may now supplement Theorem 4.3 by some one-sided implications.

THEOREM 4.4. Let $(X, Y)$ and $\left(X_{n}, Y_{n}\right), n \in \mathrm{N}$, be pairwise uniformly semitangential local martingales such that $X$ and all $X_{n}$ are conditionally symmetric. 
Then

(i) $\left\{X^{*}<\infty\right\} \subset\left\{Y^{*}<\infty\right\}$ a.s.;

(ii) $X_{n}^{*} \stackrel{P}{\rightarrow} 0 \Rightarrow Y_{n}^{*} \stackrel{P}{\rightarrow} 0$.

The same properties hold when the processes $(X, Y)$ and $\left(X_{n}, Y_{n}\right)$ are pairwise tangential semi-martingales such that $X$ and all $X_{n}$ have conditionally independent increments.

Proof. (i) Write $X=X^{\prime}+X^{\prime \prime}$ and $Y=Y^{\prime}+Y^{\prime \prime}$, where $X^{\prime}$ and $Y^{\prime}$ are the sums of all compensated jumps in $X$ and $Y$, respectively, of modulus greater than $r$, and assume that $X^{\prime}$ and $Y^{\prime}$ are strictly tangential whereas $X^{\prime \prime}$ and $Y^{\prime \prime}$ are weakly tangential. Using Theorem 4.3 (i) and Lemma 4.2 (i), and noting that $Y^{*} \leqslant Y^{\prime *}+Y^{\prime *}$, we get a.s.

$$
\begin{aligned}
\left\{X^{*}<\infty\right\} & =\left\{X^{\prime *}<\infty\right\} \cap\left\{X^{\prime \prime}<\infty\right\} \\
& =\left\{Y^{\prime *}<\infty\right\} \cap\left\{Y^{\prime \prime}<\infty\right\} \subset\left\{Y^{*}<\infty\right\} .
\end{aligned}
$$

(ii) Assume that $X_{n}^{*} \stackrel{P}{\rightarrow} 0$. Writing $X_{n}=X_{n}^{\prime}+X_{n}^{\prime \prime}$ and $Y_{n}=Y_{n}^{\prime}+Y_{n}^{\prime \prime}$ as before, we see from Lemma 4.2 (ii) that $X_{n}^{\prime *} \stackrel{P}{\rightarrow} 0$ and $X_{n}^{\prime \prime *} \stackrel{P}{\rightarrow} 0$, and so by Theorem 4.3 (ii) we have $Y_{n}^{\prime *} \stackrel{P}{\rightarrow} 0$ and $Y_{n}^{\prime \prime *} \stackrel{P}{\rightarrow} 0$, which implies $Y_{n}^{*} \stackrel{P}{\rightarrow} 0$.

To prove the last statement, use Theorem 4.2 and proceed as in the proof of Theorem 4.3.

The preceding theory leads to important principles of decoupling and symmetrization. Thus, to determine for a non-decreasing or conditionally symmetric process $X$ whether the maximum $X^{*}$ is finite or infinite, we may assume that $X$ has conditionally independent increments. By a further conditioning, we may reduce to the case of strictly independent increments, where the problem is elementary and the solution is essentially known. Under certain conditions, we may also reduce the problem for general martingales to one for martingales with symmetric jumps. Similar remarks apply to the criteria for convergence $X_{n}^{*} \stackrel{P}{\rightarrow} 0$ and associated tightness.

\section{STOCHASTIC INTEGRATION}

When applying the results of the previous sections to stochastic integrals, we need to know to what extent the tangential and other properties are preserved by stochastic integration. Recall the notation

$$
(V \cdot X)_{t}=\int_{0}^{t} V d X, \quad t \geqslant 0 .
$$


LEMMA 5.1. Consider some semi-martingales $X$ and $Y$ and a predictable process $V$, such that the stochastic integrals below exist, and in case of (ii) are even integrable.

(i) If $X$ and $Y$ are tangential, so are $V \cdot X$ and $V \cdot Y$.

(ii) If $X$ and $Y$ are centered, weakly tangential, so are $V \cdot X$ and $|V| \cdot Y$.

(iii) If $X$ and $Y$ are semi-tangential, so are $V \cdot X$ and $V \cdot Y$ for bounded $V$.

(iv) If $X$ is conditionally symmetric, so is $V \cdot X$.

(v) If $\tilde{X}$ has conditionally independent increments given $\mathcal{F}$, so has $V \cdot \tilde{X}$.

Proof. (i) Recall that if $\xi$ is a marked point process with compensator $\hat{\xi}$, and the process $V \geqslant 0$ is predictable, then $V \cdot \xi$ has compensator $V \cdot \hat{\xi}$. Further, note that if $X$ has jump point process $\xi$, then the jump point process of $-X$ is the reflection of $\xi$, whose compensator is obtained by reflection of $\hat{\xi}$. Combining those facts, we see that if the point processes $\xi$ and $\eta$ are tangential, then so are $V \cdot \xi$ and $V \cdot \eta$. For the continuous martingale component $M$ of $X$, we have $[V \cdot M]=$ $V^{2} \cdot[M]$. We also note that if $X$ has a predictable drift component $A$, then the process $V \cdot A$ is again predictable. The assertion for $X$ and $Y$ now follows easily by combination.

(ii) If $X$ and $Y$ are weakly tangential, then $[X]$ and $[Y]$ are strictly tangential, and hence so are $[V \cdot X]=V^{2} \cdot[X]$ and $[|V| \cdot Y]=V^{2} \cdot[Y]$ by part (i). Furthermore, the centering of $X$ and $Y$ is clearly preserved by stochastic integration.

(iii) If $|\Delta X| \leqslant b$ and $|V| \leqslant c$, then $|\Delta(V \cdot X)| \leqslant b c$, and similarly for $Y$. Now apply (i) and (ii).

(iv) It is enough to consider the jump point process $\xi$, where symmetry means that the compensator $\hat{\xi}$ is symmetric. The latter symmetry is clearly preserved by the integration $V \cdot \xi$ when $V \geqslant 0$, and also by a change of sign, since the reflected version of $\xi$ has the same compensator $\hat{\xi}$. The assertion now follows by combination.

(v) Conditioning on $\mathcal{F}$, we may reduce to the case where $\tilde{X}$ has independent increments and $V$ is non-random, in which case clearly $V \cdot \tilde{X}$ has again independent increments.

We may now give conditions for the validity of

$$
\left\{(V \cdot X)^{*}<\infty\right\} \subset\left\{(V \cdot Y)^{*}<\infty\right\} \text { a.s. }
$$

along with the corresponding relation with a.s. equality. This is especially useful when $X$ has conditionally independent increments, since precise criteria for the condition on the left can then be inferred from the results in Section 2.

COROLlary 5.1. Let $X$ and $Y$ be semi-martingales, where $X$ has conditionally independent increments, and let $V$ be a predictable process such that $V \cdot X$ and $V \cdot Y$ exist. Then the inclusion in (5.1) holds a.s. under each of the conditions (i) or (ii), 
(i) $X$ and $Y$ are tangential,

(ii) $X$ and $Y$ are semi-tangential, $X$ is conditionally symmetric, and $V$ is bounded, whereas a.s. equality holds in (5.1) under each of the following conditions:

(iii) $X$ and $Y$ are tangential increasing, and $V \geqslant 0$,

(iv) $X$ and $Y$ are tangential and conditionally symmetric,

(v) $X$ and $Y$ are centered, weakly tangential with bounded jumps, and $V$ is bounded.

Proof. (i) The processes $V \cdot X$ and $V \cdot Y$ are again tangential by Lemma 5.0 (i), and Lemma 5.$]$ (v) shows that $V \cdot X$ has again conditionally independent increments. Relation (5.J) now follows by Theorem 4.4 (i).

(ii) Here $V \cdot X$ and $V \cdot Y$ are again semi-tangential by Lemma 5.$]$ (iii), and Lemma 5. (iv) shows that $V \cdot X$ is again conditionally symmetric. Hence, (5.D) follows again by Theorem 4.4 (i).

(iii) Here Lemma 5 . (i) shows that $V \cdot X$ and $V \cdot Y$ are again tangential increasing, and so equality holds in (5.1) by Theorem 4.3 (i).

(iv) From Lemma 5.1 (i) and (iv) we see that $V \cdot X$ and $V \cdot Y$ are again tangential and conditionally symmetric, and so equality holds in (5.]) by Theorem 4.3 (i).

(v) Here Lemma 5 .] (ii) shows that $V \cdot X$ and $V \cdot Y$ are again centered, weakly tangential with bounded jumps, and so we have again equality in (5.1) by Theorem 4.3 (i).

By similar arguments, here omitted, we obtain sufficient conditions for the implication

$$
\left(V_{n} \cdot X_{n}\right)^{*} \stackrel{P}{\rightarrow} 0 \Rightarrow\left(V_{n} \cdot Y_{n}\right)^{*} \stackrel{P}{\rightarrow} 0,
$$

along with the corresponding equivalence.

COROLLARY 5.2. For each $n \in \mathrm{N}$, let $X_{n}$ and $Y_{n}$ be semi-martingales, where $X_{n}$ has conditionally independent increments, and let $V_{n}$ be a predictable process such that $V_{n} \cdot X_{n}$ and $V_{n} \cdot Y_{n}$ exist. Then (5.2) holds under each of the conditions (i) or (ii),

(i) the $X_{n}$ and $Y_{n}$ are tangential,

(ii) the $X_{n}$ and $Y_{n}$ are uniformly semi-tangential, the $X_{n}$ are conditionally symmetric, and the $V_{n}$ are uniformly bounded, whereas equivalence holds in (5.2) under each of the following conditions:

(iii) the $X_{n}$ and $Y_{n}$ are tangential increasing, and $V_{n} \geqslant 0$,

(iv) the $X_{n}$ and $Y_{n}$ are tangential and conditionally symmetric,

(v) the $X_{n}$ and $Y_{n}$ are centered, weakly tangential with uniformly bounded jumps, and the $V_{n}$ are uniformly bounded. 
We conclude with a decoupling property for divergence to infinity.

THEOREM 5.1. For every $n \in \mathrm{N}$, consider a T-marked point process $\xi_{n}$ on $(0, \infty)$ with compensator $\eta_{n}$ and p-thinnings $\xi_{n}^{p}$, a predictable process $V_{n}$ on $(0, \infty) \times T$, and a $\xi_{n}$-tangential process $\zeta_{n} \Perp_{\eta_{n}} V_{n}$ with conditionally independent increments. Let $\tilde{\xi}_{n}$ and $\tilde{\zeta}_{n}$ be symmetrized versions of $\xi_{n}$ and $\zeta_{n}$. Then

(i) $\xi_{n}^{p} V_{n}^{2} \stackrel{P}{\rightarrow} \infty, p \in(0,1] \Rightarrow \zeta_{n} V_{n}^{2} \stackrel{P}{\rightarrow} \infty$;

(ii) $\left(V_{n} \cdot \tilde{\xi}_{n}^{p}\right)^{*} \stackrel{P}{\rightarrow} \infty, p \in(0,1] \Rightarrow\left(V_{n} \cdot \tilde{\zeta}_{n}\right)^{*} \stackrel{P}{\rightarrow} \infty$.

Proof. (i) Put $U_{n}=V_{n}^{2}$ and $\hat{U}_{n}=U_{n} \wedge 1$. For fixed $r>0$, we introduce the optional times

$$
\tau_{n}=\inf \left\{t \geqslant 0 ;\left(\hat{U}_{n} \cdot \eta_{n}\right)_{t}>r\right\}, \quad n \in \mathrm{N} .
$$

Using Chebyshev's inequality, the conditional independence $\xi_{n}^{p} \Perp_{\xi_{n}} U_{n}$, and the compensation property of $\eta_{n}$, we get

and so

$$
\begin{aligned}
P\left\{\left(U_{n} \cdot \xi_{n}^{p}\right)_{\tau_{n}} \geqslant 1\right\} & =P\left\{\left(\hat{U}_{n} \cdot \xi_{n}^{p}\right)_{\tau_{n}} \geqslant 1\right\} \\
& \leqslant E\left(\hat{U}_{n} \cdot \xi_{n}^{p}\right)_{\tau_{n}}=p E\left(\hat{U}_{n} \cdot \xi_{n}\right)_{\tau_{n}} \\
& =p E\left(\hat{U}_{n} \cdot \eta_{n}\right)_{\tau_{n}} \leqslant p(r+1),
\end{aligned}
$$

$$
\begin{aligned}
P\left\{\eta_{n} \hat{U}_{n} \leqslant r\right\} & =P\left\{\tau_{n}=\infty\right\} \\
& \leqslant P\left\{\xi_{n}^{p} U_{n}<1\right\}+P\left\{\left(U_{n} \cdot \xi_{n}^{p}\right)_{\tau_{n}} \geqslant 1\right\} \\
& \leqslant P\left\{\xi_{n}^{p} U_{n}<1\right\}+p(r+1) .
\end{aligned}
$$

Since $\xi_{n} U_{n} \stackrel{P}{\rightarrow} \infty$, we obtain $P\left\{\eta_{n} \hat{U}_{n} \leqslant r\right\} \rightarrow 0$ as $n \rightarrow \infty$ and then $p \rightarrow 0$, and $r$ being arbitrary, we get $\eta_{n} \hat{U}_{n} \stackrel{P}{\rightarrow} \infty$. Then for every subsequence $N^{\prime} \subset \mathrm{N}$ we have $\eta_{n} \hat{U}_{n} \rightarrow \infty$ a.s. along a further subsequence $N^{\prime \prime}$. Since $\zeta_{n}$ has conditionally independent increments and intensity $\eta_{n}$, given $\left(\eta_{n}, U_{n}\right)$, Lemma 2.1$]$ (iii) yields $\zeta_{n} U_{n} \stackrel{P}{\rightarrow} \infty$, conditionally along $N^{\prime \prime}$. The corresponding unconditional property follows by dominated convergence, and the convergence extends to $\mathrm{N}$ since $N^{\prime}$ was arbitrary.

(ii) Here we put instead $\hat{V}_{n}=2 V_{n} /\left(\left|V_{n}\right| \vee 2\right)$, and consider for every $r>0$ the optional times

$$
\tau_{n}=\inf \left\{t \geqslant 0 ;\left(\hat{V}_{n}^{2} \cdot \eta_{n}\right)_{t}>r\right\}, \quad n \in \mathrm{N} .
$$

Using a BDG-inequality and proceeding as before, we get

$$
\begin{aligned}
P\left\{\left(V_{n} \cdot \tilde{\xi}_{n}^{p}\right)_{\tau_{n}}^{*} \geqslant 1\right\} & =P\left\{\left(\hat{V}_{n} \cdot \tilde{\xi}_{n}^{p}\right)_{\tau_{n}}^{*} \geqslant 1\right\} \leqslant E\left(\hat{V}_{n} \cdot \tilde{\xi}_{n}^{p}\right)_{\tau_{n}}^{* 2} \\
& =p^{2} E\left(\hat{V}_{n} \cdot \tilde{\xi}_{n}\right)_{\tau_{n}}^{* 2} \leq p^{2} E\left(\hat{V}_{n}^{2} \cdot \xi_{n}\right)_{\tau_{n}} \\
& =p^{2} E\left(\hat{V}_{n}^{2} \cdot \eta_{n}\right)_{\tau_{n}} \leqslant p^{2}(r+4),
\end{aligned}
$$


and so

$$
\begin{aligned}
P\left\{\eta_{n} \hat{V}_{n}^{2} \leqslant r\right\} & =P\left\{\tau_{n}=\infty\right\} \\
& \leqslant P\left\{\left(V_{n} \cdot \xi_{n}^{p}\right)^{*}<1\right\}+P\left\{\left(V_{n} \cdot \xi_{n}^{p}\right)_{\tau_{n}}^{*} \geqslant 1\right\} \\
& \leq P\left\{\left(V_{n} \cdot \xi_{n}^{p}\right)^{*}<1\right\}+p^{2}(r+4),
\end{aligned}
$$

which implies $\eta_{n} \hat{V}_{n}^{2} \stackrel{P}{\rightarrow} \infty$. We may now complete the proof as before, except that now we need to use Lemma 2.2 (iii).

\section{MULTIPLE STOCHASTIC INTEGRALS}

The tangential methods of Sections 3-5 will now be used to extend the existence and convergence criteria of Section 2.2 to multiple integrals $\xi_{1} \ldots \xi_{d} f$ with respect to arbitrary marked point processes. Our main conclusion is that the previous results remain valid if we replace the intensities $\mu_{k}=E \xi_{k}$ in the independence case by certain sequential compensators $\eta_{1}, \ldots, \eta_{d}$, defined as below. This essentially solves the existence and convergence problems for arbitrary non-decreasing processes.

Given some $T$-marked point processes $\xi_{1}, \ldots, \xi_{d}$ on $(0, \infty)$ adapted to a filtration $\mathcal{F}$, we take $\eta_{1}$ to be the $\mathcal{F}$-compensator of $\xi_{1}$. Continuing recursively, suppose that $\eta_{1}, \ldots, \eta_{k-1}$ have already been defined for some $k \leqslant d$. Then let $\eta_{k}$ be the compensator of $\xi_{k}$ with respect to the extended filtration

$$
\mathcal{F}_{t}^{k}=\mathcal{F}_{t} \vee \sigma\left(\eta_{1}, \ldots, \eta_{k-1}\right), \quad t \geqslant 0, k=1, \ldots, d .
$$

We may also introduce the sequentially tangential processes $\tilde{\xi}_{1}, \ldots, \tilde{\xi}_{d}$, where each $\tilde{\xi}_{k}$ is $\mathcal{F}^{k}$-tangential to $\xi_{k}$ with conditionally independent increments and intensity $\eta_{k}$, and the $\tilde{\xi}_{k}$ are conditionally independent, given $\eta_{1}, \ldots, \eta_{d}$. (The existence is clear from Theorem B.1, applied recursively, though in each step we may need to replace the underlying filtration by a suitable standard extension.)

We may now state our basic existence and convergence criteria. Here a function $f$ on $\left(\mathrm{R}_{+} \times T\right)^{d}$ is said to be tetrahedral if it is supported by the set where $t_{1}<\ldots<t_{d}$.

THEOREM 6.1. Let $\xi_{1}, \ldots, \xi_{d}$ be $\mathcal{F}$-adapted, T-marked point processes on $(0, \infty)$ with associated sequentially tangential processes $\tilde{\xi}_{1}, \ldots, \tilde{\xi}_{d}$, and similarly for $\xi_{1}^{n}, \ldots, \xi_{d}^{n}$ and $\tilde{\xi}_{1}^{n}, \ldots, \tilde{\xi}_{d}^{n}$. Then for any tetrahedral functions $f, f_{1}, f_{2}, \ldots \geqslant 0$ on $\left(\mathrm{R}_{+} \times T\right)^{d}$, we have

(i) $\xi_{1} \ldots \xi_{d} f<\infty$ a.s. $\Leftrightarrow \tilde{\xi}_{1} \ldots \tilde{\xi}_{d} f<\infty$ a.s.

(ii) $\xi_{1}^{n} \ldots \xi_{d}^{n} f_{n} \stackrel{P}{\rightarrow} 0 \Leftrightarrow \tilde{\xi}_{1}^{n} \ldots \tilde{\xi}_{d}^{n} f_{n} \stackrel{P}{\rightarrow} 0$.

When the processes $\xi_{1}, \ldots, \xi_{d}$ are independent with independent increments and intensities $\mu_{1}, \ldots, \mu_{d}$, we may define

$$
\mathcal{C}_{d}\left(\mu_{1}, \ldots, \mu_{d}\right)=\left\{f \geqslant 0 ; \xi_{1} \ldots \xi_{d} f<\infty \text { a.s. }\right\},
$$


which is essentially given by Lemma 2.4 above. Assertion (i) is then equivalent to

$$
\xi_{1} \ldots \xi_{d} f<\infty \text { a.s. } \Leftrightarrow f \in \mathcal{C}_{d}\left(\eta_{1}, \ldots, \eta_{d}\right) \text { a.s. }
$$

Similarly, we may restate part (ii) in terms of the conditions in Lemma 2.4 (ii). Both assertions follow easily from the following comparison lemma.

LEMMA 6.1. Let $\xi_{1}, \ldots, \xi_{d}$ and $\tilde{\xi}_{1}, \ldots, \tilde{\xi}_{d}$ be such as in Theorem 6.11 Then for any tetrahedral function $f \geqslant 0$ on $\left(\mathrm{R}_{+} \times T\right)^{d}$ and increasing function $\varphi \geqslant 0$ of moderate growth, we have

$$
E \varphi\left(\xi_{1} \ldots \xi_{d} f\right) \asymp E \varphi\left(\tilde{\xi}_{1} \ldots \tilde{\xi}_{d} f\right),
$$

where the domination constants depend only on $\varphi$ and $d$.

Pr o o f. The assertion for $d=1$ follows from Theorem 4.]. Now assume the statement to be true in dimension $d-1$. In $d$ dimensions, Lemma 3.5 shows that the processes $\tilde{\xi}_{2}, \ldots, \tilde{\xi}_{d}$ are sequentially tangential with respect to the filtration $\mathcal{F}^{2}$ and probability measure $P\left[\cdot \mid \eta_{1}\right]$, and so the induction hypothesis yields a.s.

$$
E\left[\varphi\left(\xi_{2} \ldots \xi_{d} g\right) \mid \eta_{1}\right] \asymp E\left[\varphi\left(\tilde{\xi}_{2} \ldots \tilde{\xi}_{d} g\right) \mid \eta_{1}\right],
$$

simultaneously for all measurable functions $g \geqslant 0$ on $\left(\mathrm{R}_{+} \times T\right)^{d-1}$. Since $f$ is tetrahedral, the partial integral $\xi_{2} \ldots \xi_{d} f$ is predictable in the remaining argument, and so the associated integrals with respect to $\xi_{1}$ and $\tilde{\xi}_{1}$ are again tangential. Using Theorem 4.11, the disintegration Theorem 6.4 in [12], the conditional independence

$$
\tilde{\xi}_{1} \Perp_{\eta_{1}}\left(\xi_{2}, \ldots, \xi_{d}, \tilde{\xi}_{2}, \ldots, \tilde{\xi}_{d}\right),
$$

and relation (6. لل with $g$ replaced by the partial integral $\mu f$, we get

$$
\begin{aligned}
E \varphi\left(\xi_{1} \ldots \xi_{d} f\right) & \asymp E \varphi\left(\tilde{\xi}_{1} \xi_{2} \ldots \xi_{d} f\right) \\
& =E E\left[\varphi\left(\mu \xi_{2} \ldots \xi_{d} f\right) \mid \eta_{1}, \tilde{\xi}_{1}\right]_{\mu=\tilde{\xi}_{1}} \\
& =E E\left[\varphi\left(\mu \xi_{2} \ldots \xi_{d} f\right) \mid \eta_{1}\right]_{\mu=\tilde{\xi}_{1}} \\
& \asymp E E\left[\varphi\left(\mu \tilde{\xi}_{2} \ldots \tilde{\xi}_{d} f\right) \mid \eta_{1}\right]_{\mu=\tilde{\xi}_{1}} \\
& =E E\left[\varphi\left(\mu \tilde{\xi}_{2} \ldots \tilde{\xi}_{d} f\right) \mid \eta_{1}, \tilde{\xi}_{1}\right]_{\mu=\tilde{\xi}_{1}} \\
& =E \varphi\left(\tilde{\xi}_{1} \tilde{\xi}_{2} \ldots \tilde{\xi}_{d} f\right),
\end{aligned}
$$

where we note that, in both approximation steps, the domination constants depend only on $\varphi$ and $d$. This completes the induction.

Pr o of of The or e m 6.1. Taking $\varphi(x)=x \wedge 1$, we see from Lemma 6.1 that, under the given conditions,

$$
E\left(\xi_{n}^{1} \ldots \xi_{n}^{d} f_{n} \wedge 1\right) \asymp E\left(\tilde{\xi}_{n}^{1} \ldots \tilde{\xi}_{n}^{d} f_{n} \wedge 1\right),
$$

which yields the stated equivalence. The assertions now follow, since $\xi_{1} \ldots \xi_{d} f$ $<\infty$ a.s. iff $\xi_{1} \ldots \xi_{d}(c f) \stackrel{P}{\rightarrow} 0$ as $c \rightarrow 0$, and similarly for $\tilde{\xi}_{1} \ldots \tilde{\xi}_{d} f$. 
The previous results simplify when the processes $\xi_{1}, \ldots, \xi_{d}$ are independent. Though the independence may not carry over to the associated compensators $\eta_{1}, \ldots, \eta_{d}$, we do have a simple criterion in terms of some mutually independent random measures $\tilde{\eta}_{k} \stackrel{d}{=} \eta_{k}$.

COROllary 6.1. Consider some independent, adapted, T-marked point processes $\xi_{1}, \ldots, \xi_{d}$ on $(0, \infty)$, having compensators $\eta_{1}, \ldots, \eta_{d}$ with mutually independent copies $\tilde{\eta}_{1}, \ldots, \tilde{\eta}_{d}$, and similarly for processes indexed by $n$. Then for any functions $f, f_{1}, f_{2}, \ldots \geqslant 0$ on $\left(\mathrm{R}_{+} \times T\right)^{d}$,

(i) $P\left\{\xi_{1} \ldots \xi_{d} f<\infty\right\}=P\left\{f \in \mathcal{C}_{d}\left(\tilde{\eta}_{1}, \ldots, \tilde{\eta}_{d}\right)\right\}$;

(ii) $\xi_{1}^{n} \ldots \xi_{d}^{n} f_{n} \stackrel{P}{\rightarrow} 0 \Leftrightarrow \tilde{\xi}_{1}^{n} \ldots \tilde{\xi}_{d}^{n} f_{n} \stackrel{P}{\rightarrow} 0$.

Proof. (i) Consider some independent copies $\xi_{1}^{\prime}, \ldots, \xi_{d_{\sim}}^{\prime}$ of $\xi_{1}, \ldots, \xi_{d}$ and $\tilde{\eta}_{1}, \ldots, \tilde{\eta}_{d}$ of $\eta_{1}, \ldots, \eta_{d}$, and conditionally on $\tilde{\eta}_{1}, \ldots, \tilde{\eta}_{d}$, let $\tilde{\xi}_{1}^{\prime}, \ldots, \tilde{\xi}_{d}^{\prime}$ be independent of $\xi_{1}, \ldots, \xi_{d}$ and mutually independent with independent increments and intensities $\tilde{\eta}_{1}, \ldots, \tilde{\eta}_{d}$. Then Theorem 4.3 (i) yields

$$
\begin{aligned}
P\left\{\xi_{1} \ldots \xi_{d} f<\infty\right\} & =P\left\{\xi_{1} \xi_{2}^{\prime} \ldots \xi_{d}^{\prime} f<\infty\right\} \\
& =P\left\{\tilde{\xi}_{1} \xi_{2}^{\prime} \ldots \xi_{d}^{\prime} f<\infty\right\} \\
& =P\left\{\tilde{\xi}_{1}^{\prime} \xi_{2} \ldots \xi_{d} f<\infty\right\} .
\end{aligned}
$$

Since $\tilde{\xi}_{1}^{\prime} \Perp\left(\xi_{2}, \ldots, \xi_{d}\right)$, we may continue recursively in $d$ steps to get

$$
P\left\{\xi_{1} \ldots \xi_{d} f<\infty\right\}=P\left\{\tilde{\xi}_{1}^{\prime} \ldots \tilde{\xi}_{d}^{\prime} f<\infty\right\},
$$

which is equivalent to the asserted relation.

(ii) Proceeding as before, we get for any non-decreasing function $\varphi \geqslant 0$ of moderate growth

$$
E \varphi\left(\xi_{1}^{n} \ldots \xi_{d}^{n} f_{n}\right) \asymp E \varphi\left(\tilde{\xi}_{1}^{n} \ldots \tilde{\xi}_{d}^{n} f_{n}\right)
$$

where the domination constants depend only on $\varphi$ and $d$. Now let $n \rightarrow \infty$.

The signed case is more difficult, since existence is then defined by a Cauchy criterion for the final values, whereas the tangential comparison is stated in terms of the maximum values. This is why we need the tangent processes $\tilde{\xi}_{1}, \ldots, \tilde{\xi}_{d}$ to satisfy the maximum property in Corollary 2.3. Another complication is that a possible martingale property of $\xi_{1}, \ldots, \xi_{d}$ is not preserved, in general, by the sequential tangent processes $\tilde{\xi}_{1}, \ldots, \tilde{\xi}_{d}$. Here we give only the following one-sided result:

THEOREM 6.2. Let $\xi_{1}, \ldots, \xi_{d}$ be signed, marked point processes, such that the maximum property holds for the associated sequential tangent processes $\tilde{\xi}_{1}, \ldots, \tilde{\xi}_{d}$, and fix any measurable functions $f, f_{1}, f_{2}, \ldots$ Then

(i) $\tilde{\xi}_{1} \ldots \tilde{\xi}_{d} f$ exists $\Rightarrow \xi_{1} \ldots \xi_{d} f$ exists;

(ii) $\tilde{\xi}_{1} \ldots \tilde{\xi}_{d} f_{n} \stackrel{P}{\rightarrow} 0 \Rightarrow \xi_{1} \ldots \xi_{d} f_{n} \stackrel{P}{\rightarrow} 0$.

In (ii) it is understood that the integrals on the left exist. 
Pro o f. First we prove (ii) for functions of bounded supports. Putting $\varphi(x)=$ $|x| \wedge 1$, we may write the assertion as

$$
E \varphi\left(\tilde{\xi}_{1} \ldots \tilde{\xi}_{d} f_{n}\right) \rightarrow 0 \Rightarrow E \varphi\left(\xi_{1} \ldots \xi_{d} f_{n}\right) \rightarrow 0,
$$

which we prove by induction. For $d=1$, the maximum property yields

$$
E \varphi\left(\tilde{\xi} f_{n}\right) \rightarrow 0 \Rightarrow E \varphi\left(\left(f_{n} \cdot \tilde{\xi}\right)^{*}\right) \rightarrow 0,
$$

and so, by Theorem 4.2,

$$
E \varphi\left(\xi f_{n}\right) \leqslant E \varphi \circ\left(f_{n} \cdot \xi\right)^{*} \leq E \varphi \circ\left(f_{n} \cdot \tilde{\xi}\right)^{*} \rightarrow 0 .
$$

Assuming the truth in dimension $d-1$, we turn to the $d$-dimensional case. Using Lemma 3.5, we get as before

$$
E\left[\varphi\left(\tilde{\xi}_{2} \ldots \tilde{\xi}_{d} g_{n}\right) \mid \eta_{1}\right] \rightarrow 0 \quad \Rightarrow \quad E\left[\varphi\left(\xi_{2} \ldots \xi_{d} g_{n}\right) \mid \eta_{1}\right] \rightarrow 0
$$

outside a fixed $P$-null set. Now suppose that $E \varphi\left(\tilde{\xi}_{1} \ldots \tilde{\xi}_{d} f_{n}\right) \rightarrow 0$, so that

$$
E E\left[\varphi\left(\mu \tilde{\xi}_{2} \ldots \tilde{\xi}_{d} f_{n}\right) \mid \eta_{1}\right]_{\mu=\tilde{\xi}_{1}} \rightarrow 0
$$

by the disintegration theorem. Then for every subsequence $N^{\prime} \subset \mathrm{N}$ we have

$$
E\left[\varphi\left(\mu \tilde{\xi}_{2} \ldots \tilde{\xi}_{d} f_{n}\right) \mid \eta_{1}\right]_{\mu=\tilde{\xi}_{1}} \rightarrow 0 \text { a.s. }
$$

along a further subsequence $N^{\prime \prime} \subset N^{\prime}$, which implies

$$
E\left[\varphi\left(\mu \xi_{2} \ldots \xi_{d} f_{n}\right) \mid \eta_{1}\right]_{\mu=\tilde{\xi}_{1}} \rightarrow 0 \text { a.s. }
$$

by (6.2). By dominated convergence and the disintegration theorem we get $E \varphi\left(\tilde{\xi}_{1} \xi_{2} \ldots \xi_{d} f_{n}\right) \rightarrow 0$, and so by Lemma $5.1(\mathrm{v})$, Theorem 4.2 , and the maximum property of $\tilde{\xi}_{1}$, we have along $N^{\prime \prime}$

$$
\begin{aligned}
E \varphi\left(\xi_{1} \ldots \xi_{d} f_{n}\right) & \leqslant E \varphi \circ\left(\left(\xi_{2} \ldots \xi_{d} f_{n}\right) \cdot \xi_{1}\right)^{*} \\
& \leq E \varphi \circ\left(\left(\xi_{2} \ldots \xi_{d} f_{n}\right) \cdot \tilde{\xi}_{1}\right)^{*} \rightarrow 0
\end{aligned}
$$

(cf. Corollary 5.2 (i)). Since $N^{\prime}$ was arbitrary, this completes the induction. We may now proceed to the general case.

(i) Suppose that $\tilde{\xi}_{1} \ldots \tilde{\xi}_{d} f$ exists, and consider any functions $f_{1}, f_{2}, \ldots$ with bounded supports and $\left|f_{n}\right| \leqslant|f|$ such that $f_{n} \rightarrow f$. Then $\tilde{\xi}_{1} \ldots \tilde{\xi}_{d} f_{n} \stackrel{P}{\rightarrow} \tilde{\xi}_{1} \ldots \tilde{\xi}_{d} f$, and so the sequence of integrals $\tilde{\xi}_{1} \ldots \tilde{\xi}_{d} f_{n}$ is Cauchy in probability. By the special case of (ii), so is the sequence $\xi_{1} \ldots \xi_{d} f_{n}$, which then converges to some limiting random variable. Considering alternating sequences of the form $f_{1}, g_{1}, f_{2}, g_{2}, \ldots$, 
we see that the limit is a.s. independent of the choice of sequence $\left(f_{n}\right)$, and hence can be used to define the integral $\xi_{1} \ldots \xi_{d} f$.

(ii) Suppose that $\tilde{\xi}_{1} \ldots \tilde{\xi}_{d} f_{n} \stackrel{P}{\rightarrow} 0$. Then for each $n$ we may choose some functions $f_{n k}$ with bounded supports and $\left|f_{n k}\right| \leqslant\left|f_{n}\right|$ such that $f_{n k} \rightarrow f_{n}$ as $k \rightarrow \infty$. By (i) and linearity, we get

$$
\tilde{\xi}_{1} \ldots \tilde{\xi}_{d}\left(f_{n}-f_{n k}\right) \stackrel{P}{\rightarrow} 0, \quad \xi_{1} \ldots \xi_{d}\left(f_{n}-f_{n k}\right) \stackrel{P}{\rightarrow} 0 .
$$

Writing $\psi(x)=|x| \wedge 1$, we may choose $k=k_{n}$ so large that the functions $f_{n}^{\prime}=$ $f_{n, k_{n}}$ satisfy

$$
E \psi\left(\tilde{\xi}_{1} \ldots \tilde{\xi}_{d}\left(f_{n}-f_{n}^{\prime}\right)\right) \vee E \psi\left(\xi_{1} \ldots \xi_{d}\left(f_{n}-f_{n}^{\prime}\right)\right)<n^{-1} .
$$

Then

$$
\tilde{\xi}_{1} \ldots \tilde{\xi}_{d} f_{n}^{\prime}=\tilde{\xi}_{1} \ldots \tilde{\xi}_{d} f_{n}-\tilde{\xi}_{1} \ldots \tilde{\xi}_{d}\left(f_{n}-f_{n}^{\prime}\right) \stackrel{P}{\rightarrow} 0,
$$

and so, by (6.3) and the special case of (ii),

$$
\xi_{1} \ldots \xi_{d} f_{n}=\xi_{1} \ldots \xi_{d} f_{n}^{\prime}+\xi_{1} \ldots \xi_{d}\left(f_{n}-f_{n}^{\prime}\right) \stackrel{P}{\rightarrow} 0
$$

Acknowledgments. I am grateful for the careful reading of a referee, which led to many improvements of the presentation.

\section{REFERENCES}

[1] D. L. Burkholder, B. J. Davis, and R. F. Gundy, Integral inequalities for convex functions of operators on martingales, in: Proceedings of the Sixth Berkeley Symposium on Mathematical Statistics and Probability, Vol. 2, Univ. of California Press, 1972, pp. 223-240.

[2] B. Grigelionis, Martingale characterization of stochastic processes with independent increments, Litovsk. Mat. Sb. 17 (1977), pp. 75-86.

[3] P. Hitchenko, Comparison of moments for tangent sequences of random variables, Probab. Theory Related Fields 78 (1988), pp. 223-230.

[4] K. Itô, Differential equations determining a Markov process, J. Pan-Japan Math. Coll. 1077 (1942).

[5] K. Itô, Multiple Wiener integral, J. Math. Soc. Japan 3 (1951), pp. 157-169.

[6] J. Jacod, Multivariate point processes: Predictable projection, Radon-Nikodym derivative, representation of martingales, Z. Wahrsch. Verw. Gebiete 31 (1975), pp. 235-253.

[7] J. Jacod, Une généralisation des semimartingales: Les processus admettant un processus à accroissements indépendants tangent, in: Séminaire de Probabilités XVIII, Lecture Notes in Math., Vol. 1059, Springer, Berlin 1984, pp. 91-118.

[8] J. Jacod and H. Sadi, Processus admettant un processus à accroissements indépendants tangent: cas général, in: Séminaire de Probabilités XXI, Lecture Notes in Math., Vol. 1247, Springer, Berlin 1987, pp. 479-514.

[9] J. Jacod and A. N. Shiryaev, Limit Theorems for Stochastic Processes, Springer, Berlin 1987.

[10] A. Jakubowski, Principle of conditioning in limit theorems for sums of random variables, Ann. Probab. 14 (1986), pp. 902-915. 
[11] O. Kallenberg, Some uses of point processes in multiple stochastic integration, Math. Nachr. 151 (1991), pp. 7-31.

[12] O. Kallenberg, Foundations of Modern Probability, second edition, Springer, New York 2002.

[13] O. Kallenberg, Some failures, problems, and conjectures from a lifetime in probability, Lecture at the "Kallenberg Symposium," Mittag-Leffler Institute, Djursholm, Sweden, 2013. http://www.math.uni-frankfurt.de/ ismi/Kallenberg_symposium/Presentations/Kallenberg.pdf

[14] O. Kallenberg, Random Measures, Theory and Applications, Springer, New York 2017.

[15] O. Kallenberg and J. Szulga, Multiple integration with respect to Poisson and Lévy processes, Probab. Theory Related Fields 83 (1989), pp. 101-134.

[16] W. Krakowiak and J. Szulga, Random multilinear forms, Ann. Probab. 14 (1986), pp. 955-973.

[17] W. Krakowiak and J. Szulga, A multiple stochastic integral with respect to a strictly pstable random measure, Ann. Probab. 16 (1988), pp. 764-777.

[18] S. Kwapień and W. A. Woyczyński, Double stochastic integrals, random quadratic forms and random series in Orlicz spaces, Ann. Probab. 15 (1987), pp. 1072-1096.

[19] S. Kwapień and W. A. Woyczyński, Tangent sequences of random variables: Basic inequalities and their applications, in: Proceedings of Conference on Almost Everywhere Convergence in Probability and Ergodic Theory, G. A. Edgar and L. Sucheston (Eds.), Academic Press, New York 1989, pp. 237-265.

[20] S. Kwapień and W. A. Woyczyński, Semimartingale integrals via decoupling inequalities and tangent processes, Probab. Math. Statist. 12 (1991), pp. 165-200.

[21] S. Kwapień and W. A. Woyczyński, Random Series and Stochastic Integrals: Single and Multiple, Birkhäuser, Boston 1992.

[22] T. R. McConnell and M. S. Taqqu, Decoupling inequalities for multilinear forms in independent symmetric random variables, Ann. Probab. 14 (1986), pp. 943-954.

[23] V. H. de la Peña and E. Giné, Decoupling: From Dependence to Independence, Springer, New York 1999.

[24] V. V. Petrov, Limit Theorems of Probability Theory: Sequences of Independent Random Variables, Clarendon Press, Oxford 1995.

[25] J. Rosiński and W. A. Woyczyński, On Itô stochastic integration with respect to p-stable motion: Inner clock, integrability of sample paths, double and multiple integrals, Ann. Probab. 14 (1986), pp. 271-286.

[26] D. Surgailis, On the multiple stable integral, Z. Wahrsch. Verw. Gebiete 70 (1985), pp. 621632.

[27] J. Zinn, Comparison of martingale difference sequences, in: Probability in Banach Spaces V, Lecture Notes in Math., Vol. 1153, Springer, Berlin 1986, pp. 453-457.

Olav Kallenberg

Auburn University

Auburn, Alabama 36849, USA

221 Parker Hall

E-mail:kalleoh@auburn.edu

Received on 1.4.2015;

revised version on 18.2.2017 